\title{
On the vortical structure in a plane impinging jet
}

\author{
By J. SAKAKIBARA ${ }^{1}$, K. HISHIDA ${ }^{2}$ \\ AND W. R. C. PHILLIPS ${ }^{3}$ \\ ${ }^{1}$ Institute of Engineering Mechanics and Systems, University of Tsukuba, Tsukuba 305-8573, Japan \\ ${ }^{2}$ Department of System Design Engineering, Keio University, Yokohama 223-8522, Japan \\ ${ }^{3}$ Department of Theoretical and Applied Mechanics \\ University of Illinois at Urbana-Champaign, Urbana, IL 61801-2935, USA
}

(Received 17 December 1997 and in revised form 20 November 2000)

The vortical structure of a plane impinging jet is considered. The jet was locked both in phase and laterally in space, and time series digital particle image velocimetry measurements were made both of the jet exiting the nozzle and as it impinged on a perpendicular wall. Iso-vorticity and iso- $\lambda_{2}$ surfaces coupled with critical point theory were used to identify and clarify structure. The flow near the nozzle was much as observed in mixing layers, where the shear layer evolves into spanwise rollers, only here the rollers occurred symmetrically about the jet midplane. Accordingly the rollers were seen to depict spanwise perturbations with the wavelength of flutes at the nozzle edge and were connected, on the same side of the jet, with streamwise 'successive ribs' of the same wavelength. This wavelength was 0.71 of the distance between rollers and, contrary to some experiments in mixing layers, did not double when the rollers paired. Structures not reported previously but evident here with iso-vorticity, $\lambda_{2}$ and critical point theory are 'cross ribs', which extend from the downstream side of each roller to its counterpart across the symmetry plane; their spanwise periodic spacing exceeds that of successive ribs by a factor of three. Cross ribs stretch because of the diverging flow as the rollers approach the wall and move apart, causing the vorticity within them to intensify. This process continues until the cross ribs reach the wall and merge with 'wall ribs'. Wall ribs remain near the wall throughout the cycle and are composed of vorticity of the same sign as the cross ribs, but the intensity level of the vorticity within them is cyclic. Details of the expansion of fluid elements, evaluated from the rate of strain tensor, revealed that both cross and successive ribs align with the principal axis and that the vorticity comprising them is continuously amplified by stretching. It is further shown, by appeal to the production terms of the phase-averaged vorticity equation, that wall ribs are sustained by merging and stretching rather than reorientation of vorticity. Moreover production of vorticity is a maximum when cross and wall ribs merge and is greatest near the symmetry plane of the jet. The demise of successive ribs on the other hand occurs away from the symmetry plane and would appear to be less important dynamically than cross ribs merging with wall ribs.

\section{Introduction}

This paper is concerned with a plane jet that impinges on a flat rigid plate set perpendicular to the flow; and of particular interest is the large-scale vorticity field that arises near the plate in the vicinity of the jet's axis of symmetry. It is in this 
region, often referred to as the stagnation region but more appropriately denoted the impingement region, that mean stream-surfaces diverge about a stagnating mean stream-surface. It is also in this region that turbulent kinetic energy is produced by both the mean strain normal to the plate and the normal component of the Reynolds stress tensor. The augmentation of turbulence in the impingement region gives rise to a substantial increase in the stagnation-line heat or mass transfer rate and is consequently used as a high-performance technique for cooling, heating or drying a surface (Gardon \& Akfirat 1965).

Looney \& Walsh (1984) give a review of experimental studies of impinging jets and note that the first complete study, in the sense of providing both mean-flow and turbulence characteristics, was by Gutmark, Wolfshtein \& Wygnanski (1978). Accordingly the first indications of flow structure were by Yokobori, Kasagi \& Hirata (1977), who observed columnar vortices close to the plate and aligned with the flow over the plate. Using hydrogen-bubble flow visualization techniques, they revealed that the vortices extended across the symmetry plane and that the spanwise spacing of the vortices not only scaled with nozzle width but could be controlled by events upstream, specifically by the lateral placement of a thin wire at the nozzle exit.

The importance of vortices perpendicular to the symmetry plane had earlier been foreseen and analyzed in the presence of stagnations point or Hiemenz flow (see Batchelor 1967, §5.5) by Sutera, Maeder \& Kestin (1963). They found that the vorticity of vortex filaments with a spanwise spacing greater than 2.6 times the Hiemenz stagnation-point boundary layer thickness, is amplified by the mean strain as the filaments advect towards the wall. Indeed, filaments with small intensity present in the oncoming flow, can reach the boundary layer with a greatly enhanced intensity and induce substantial three-dimensional effects therein. These findings led Sadeh \& Brauer (1980) to construct an experiment in which a cylinder was placed in a uniform flow in which columnar vortices had been embedded at right angles to the cylinder. Their study confirmed that the vortices were stretched and selectively amplified as they traversed the impingement region.

Further details of events in the symmetry plane of the impingement region were determined by Sakakibara, Hishida \& Maeda $(1995,1997)$, who used digital particle image velocimetry (DPIV) to measure the instantaneous velocity field and its time variation. They observed not only wall vortices as seen by Yokobori et al., but a recurring presence of concentrations of streamwise (in the sense of flow over the plate) vorticity that advected towards and merged with the wall vortices.

But while the presence and importance of streamwise vortices in the impingement region is well established, what is not known is the mechanism by which they are sustained. Of course upstream events doubtless play a role: indeed Didden \& Ho (1985) show that the ring vortices periodically generated in the shear layer of an axisymmetric impinging jet induce unsteady separation in the impingment region, a feature further explored by Fox et al. (1993). But the evolution of coherent structures in a plane jet (Antonia et al. 1986), not to mention plane impinging jet, are not nearly so well documented as say those in an axisymmetric impinging jet or in a plane mixing layer. The object of the present work therefore was to determine not only the phase evolution of the three-dimensional vorticity field in the impingment region, but also the structure of the plane jet.

Of course plane and axisymmetric jets have long been known to be susceptible to phase locking (Perry \& Lim 1978) and so in order to obtain such information, the jet was phase-locked by temporal flow perturbations and, in the vein of plane 


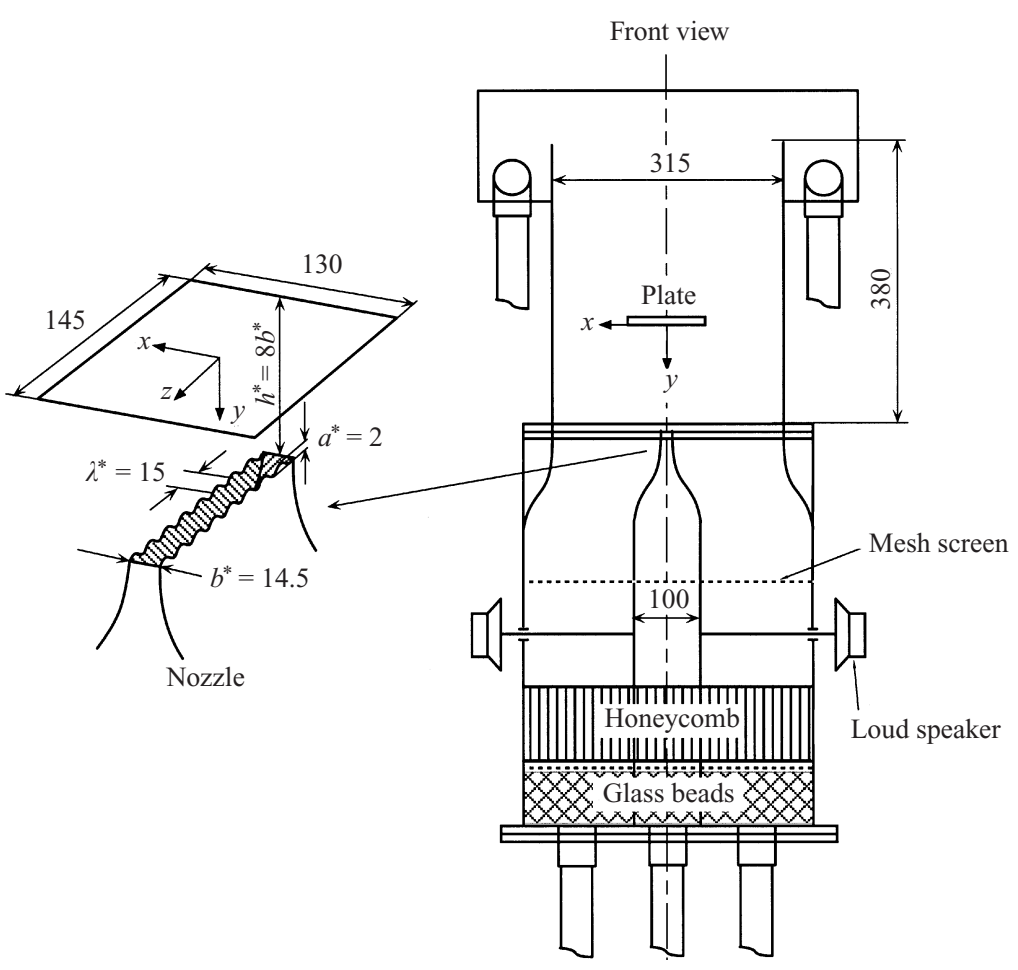

FIGURE 1. Schematic of the experimental apparatus. All dimensions are in mm.

mixing layers (Lasheras \& Choi 1988), spatially locked by a fluted nozzle. Such locking enabled three-dimensional, three-component phase-averaged velocity fields to be determined from a montage of instantaneous two-dimensional DPIV slices of the displacement field. Structural features in the flow were, for the most part, identified with the aid of iso-vorticity surfaces. However since such surfaces lack clarity in strong shear, they were supplemented by instantaneous streamlines and critical point theory in two planes of symmetry, coupled with Galilean invariant iso- $\lambda_{2}$ surfaces (Jeong \& Hussain 1995).

We find that the jet shear layer evolves in much the same manner as a plane mixing layer by developing into spanwise rollers, although here by design the rollers occur symmetrically about the midplane of the jet. Accordingly, rollers on the same side of the jet are connected with rib vortices, or 'successive ribs'. New, however, are cross rib vorticies which connect rollers across the symmetry plane. Both structures are evident with iso-vorticity surfaces, but $\lambda_{2}$ together with instantaneous streamlines and critical point theory render a less biased picture. The (spanwise periodic) spacing of cross ribs exceeds that of successive ribs by a factor of three. But it is cross rib vorticity that intensifies through stretching as the rollers progress through the impingment region and cross rib vorticity that periodically merges with the ever present rib vortices at the wall.

The paper is organized as follows. We begin (in §2) with a description of the experimental apparatus and techniques used to acquire the data. Basic features of the time-mean and phase-averaged flow are given in $\S 3$, while details of the evolution of successive, cross and wall ribs are given in $\$ \S 4,5$. The work is discussed and summarized in $\S 6$. 


\section{Experimental procedure}

\subsection{Flow facility}

The experiment consisted of a vertical planar jet which impinged on a finite plate set normal to the flow direction. The jet was in the presence of a weak coflowing stream and water was used as the working fluid. The water jet facility, shown schematically in figure 1, consisted of a settling chamber and contraction nozzle for both the jet and coflowing stream, each of which fed into a rectangular channel $(380 \times 315 \times 150 \mathrm{~mm}$, in height, width and span). The settling chambers for both the jet and surrounding flow were composed of layers of glass beads, honeycomb and fine mesh screens. Both flows then passed into smooth contraction nozzles and issued vertically upward. The nozzle exit had a width $b^{*}=14.5 \mathrm{~mm}$ and an aspect ratio of 10.3 .

The coflowing stream acted to eliminate recirculating flow and ensure a supply of fluid for jet entrainment; it was supplied with a uniform velocity of $0.05 V_{0}^{*}$, where $V_{0}^{*}=122 \mathrm{~mm} \mathrm{~s}^{-1}$ was the peak mean velocity at the nozzle exit. At a spacing $h^{*}$ from the jet exit, the jet impinged on a horizontal plate. The plate was made from transparent Plexiglas and measured $145 \times 130 \mathrm{~mm}$. Moreover, it was aligned with its longer side in the direction of the longer side of the nozzle and arranged so that the normal to its centre of gravity intersected the centre of gravity of the nozzle. The jet Reynolds number $R e=b^{*} V_{0}^{*} / v^{*}$ was held constant at 2000 . Lastly, the nozzle-to-plate spacing was set to $h^{*} / b^{*}=8$. This value was chosen because it ensures the maximum heat transfer rate at the stagnation point (Gardon \& Akfirat 1965) and would therefore appear to be the most interesting case to study.

\subsection{Nomenclature and coordinate system}

We use * to denote dimensional quantities and employ a rectangular Cartesian coordinate system $(x, y, z)$ with unit vectors $(\boldsymbol{i}, \boldsymbol{j}, \boldsymbol{k})$, and respective instantaneous velocity $(u, v, w)$ and vorticity $\left(\omega_{x}, \omega_{y}, \omega_{z}\right)$ components. Accordingly, time-mean averages $(\S 2.5)$ are written $(U, V, W) \equiv(\bar{u}, \bar{v}, \bar{w})$, and phase averages $(\langle u\rangle,\langle v\rangle,\langle w\rangle)$. Here, the coordinates have been referred to $b^{*}$, velocity to $V_{0}^{*}=V^{*}\left(0, h^{*}, 0\right)$ and the vorticity to $V_{0}^{*} / b^{*}$. Note, however, that while it is customary in studies of jets and boundary layers to set the $x$-coordinate streamwise, the jet and plate boundary layer are here orthogonal. Since the bulk of the paper is concerned with flow near the plate, we view events from the reference frame of the plate. We thus place the origin at the geometrical centre of the plate, align $x$ with the direction of mean flow over the plate and set $y$ normal to the plate (i.e. vertical, positive downwards), as shown in figure 1. Finally, for convenience when discussing events near the nozzle, we shall use the shifted coordinate $Y=h^{*} / b^{*}-y=h-y$.

\subsection{Phase-locking}

In order to phase-lock large-scale structures in the jet, the streamwise velocity was perturbed by oscillating the spanwise walls of the jet nozzle by two loudspeakers. Specifically, the perturbations introduced small oscillations in the volume flux of the inner settling chamber. Since the settling chamber was constructed from $1 \mathrm{~mm}$ thickness aluminium, this was achieved by connecting each sidewall of the inner settling chamber to the cone of a loudspeaker by metal rods; the speakers were of course mounted outside the outer settling chamber. The wave form $F^{*}\left(t^{*}\right)$ used to drive the speakers was generated by an IBM AT compatible computer with a D/A converter board and was supplied to the speakers after being low-pass filtered and 
amplified; it was constructed from two sine waves as

$$
F^{*}\left(t^{*}\right)=A_{1}^{*} \sin 2 \pi f_{1}^{*} t^{*}+A_{2}^{*} \sin \left(2 \pi f_{2}^{*} t^{*}+\phi_{P}\right) .
$$

Here $t^{*}$ is time and the fundamental frequency was set to $f_{1}^{*}=3.02 \mathrm{~Hz}$, which was the natural roll-up frequency of the jet, yielding a Strouhal number $f_{1}=b^{*} f_{1}^{*} / V_{0}^{*}=$ 0.36 ; the subharmonic frequency was set to $f_{2}^{*}=f_{1}^{*} / 2$ (see below).

The rate at which the mixing layer grows is affected by the phase lag $\phi_{P}$ between the two waves, being a minimum when $\phi_{P}=n \pi(n=0,1,2, \ldots)$ and a maximum when $\phi_{P}=(2 n+1) \pi / 2$ (Inoue 1992). Here we sought an intermediate growth rate and thus set $\phi_{P}=3 \pi / 4$; see also $\S 3.1$. The amplitudes of each of the waves, $A_{1}^{*}$ and $A_{2}^{*}$, were set at the minimum possible for locking both the phase and the amplitude of the structures identically. It transpired that the initial roll-up of the shear layer was locked when the r.m.s. turbulence intensity (which includes both coherent and non-coherent turbulence) at the nozzle exit due to forcing reached $0.009 V_{0}^{*}$.

Signals fed to facing speakers were in phase so that wall perturbations were symmetrical. Moreover, rather than basing the phase angle $\phi$ on the fundamental, it was instead based on the cycle of the subharmonic period $1 / f_{2}^{*}$, as $\phi=2 \pi f_{2} t+\phi_{P}$, where $t=t^{*} V_{0}^{*} / b^{*}$. This was done because we need to know the phase angle after the rollers (in the shear layer) have paired and such knowledge is not forthcoming if the excitation frequency is solely $f_{1}^{*}$. By adding $f_{2}^{*}$ to $f_{1}^{*}$, however, we can determine the phase angle of $f_{2}^{*}$. Accordingly, the phase average was taken with period $1 / f_{2}^{*}$.

In addition to the temporal perturbation to the jet, a spatially periodic perturbation was introduced at the exit of the nozzle to fix the spanwise location of any three-dimensional structure that subsequently formed downstream. Specifically, the (spanwise) walls of the nozzle were fluted with sinusoidal indentations of wavelength $\Lambda^{*}$ and amplitude $a^{*}$, thereby perturbing the streamwise extent of the nozzle. The indentations were similar to those used by Lasheras \& Choi (1988, henceforth referred to as LC), who studied a plane mixing layer. They suggest that the effect of each flute is to cause a sinusoidal spanwise displacement of the geometrical origin of the mixing layer, thus producing a periodic axial disturbance in the shedding vortex sheet. Prior experiments in the present apparatus indicated that the naturally occurring spanwise wavelength of the shear layer was $\Lambda^{*}=b^{*} \Lambda=15 \mathrm{~mm}$, and we chose to excite that wavelength, although LC note that virtually any spanwise wavelength can be excited. The amplitude of the flutes was chosen to be $2 \mathrm{~mm}$, a value similar to that used by LC. This amplitude was approximately five times the momentum thickness of the boundary layer at the nozzle exit.

\subsection{Data acquisition and reduction}

The data acquisition system was similar to that used and described by Sakakibara et al. (1997) in which water was marked with tracer particles $(10 \mu \mathrm{m}$ polyethylene, Sumitomo-seika HE-5023) and illuminated by a $4 \mathrm{~W}$ Ar-ion laser light sheet. Velocity fields were then measured using a custom-made digital particle image velocimetry (DPIV) system. The pulse width and the interval of the laser sheet were controlled synchronously with a CCD camera $(768 \times 494$ pixels, Sony XC-75). Synchronized stroboscopic illuminations were generated by an acousto-optical modulator cell (Hoya A-160) chopping the laser beam. Video signals from the CCD camera were recorded on a computer controllable VCR (NTSC S-VHS, Panasonic AG-5700) and a bar-code field number was superimposed onto each video field to tag each image frame. Lastly, in order to memorize the phase angle of the forcing signal at the time when each 

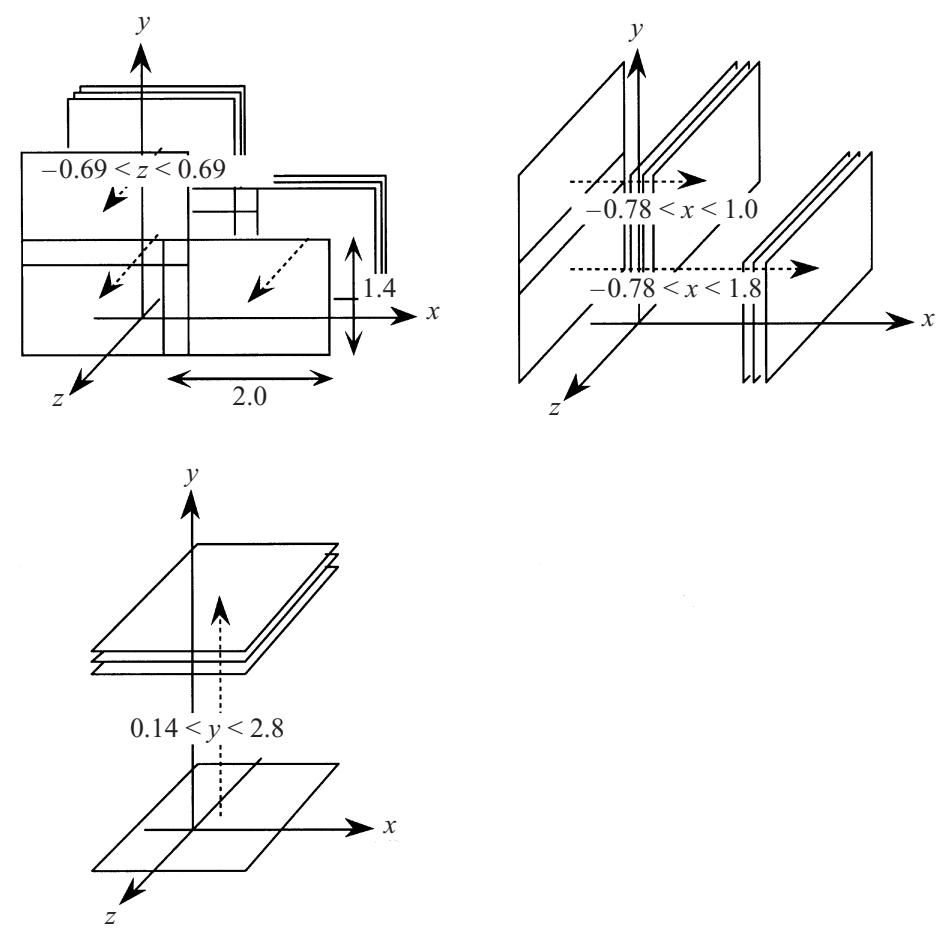

FIGURE 2. Schematic of the laser lighted measuring planes.

image was captured, the field number was recorded together with a value of the phase angle for every video image by a PC.

Velocity vectors were computed from recorded images by an IBM AT compatible computer and Texas Instruments TMS320C31 digital signal processor (DSP), which accelerates the calculation of the cross-correlation function. Fully automated image processing using thousands of images was done sequentially. Typically, 80 planes of velocity vectors could be obtained in a one hour period.

In order to obtain the phase-averaged three-dimensional distribution of the three components of vorticity by DPIV, which in fact can measure only the two-dimensional distribution of one-component of vorticity, many slices within a flow volume were measured. This volume contained $33 x, y, 32 y, z$ and $20 x, z$ measuring planes in the impingement region and $16 x, y$ measuring planes in the near nozzle region, each obtained by shifting the light plane in $2 \mathrm{~mm}$ intervals. The size of each of the measuring planes was approximately $20 \times 30 \mathrm{~mm}^{2}$ and, as shown schematically in figure 2; the planes were arranged to map out an L-shaped volume.

Prior to running the experiment, the image coordinates for each measuring plane were calibrated in terms of physical coordinates. This was done with three orthogonal laser beams positioned (by a traversing mechanism) to intersect at a known physical point in each measuring plane; a PC then recorded each point in image coordinates. One thousand instantaneous and time-series two-dimensional velocity distributions with $1 / 20 \mathrm{~s}$ time interval were obtained in each measuring plane. These were then used for calculating the statistics and phase-averaged properties.

Errors in data reduction arise from two sources, namely limitations in the resolution of particle displacements and, since we are phase averaging, downstream variations 
in phase known as jitter. The latter is particularly evident well downstream of the excitation source and errors arising from it are discussed by Hayakawa \& Hussain (1989). Such errors were not thought to be major in the present work, however, in part because the plate is relatively close to the excitation source, but also because instantaneous maps of vorticity reveal the same structural features at the same location as the phase-averaged plots. Indeed the largest error in data reduction originated from the evaluation of particle displacements, which were used to determine the velocity. This in turn was affected by the size of the interrogation area, which was $2 \times 1 \times 1 \mathrm{~mm}$. An error analysis of the data indicated that the instantaneous velocity could be determined with a $95 \%$ confidence level to within $\pm 5 \mathrm{~mm} \mathrm{~s}^{-1}$. Of course the resolution of the CCD camera (about $40 \mu \mathrm{m}$ per pixel) well exceeds the $10 \mu \mathrm{m}$ tracer particles. However the particle size on an image is typically 2 pixels owing to blurring caused by particles not completely within the $2 \mathrm{~mm}$ thick laser sheet. The diffraction limit, which is about $5 \mu \mathrm{m}$, does not play a significant role in the blurring.

\subsection{Averaging}

Time-mean $\overline{(\cdot)}$ and root-mean statistical properties of any instantaneous tensor property $\Phi(\boldsymbol{x}, t)$ were determined by taking a sufficiently long time average $T$ of $\Phi$ as

$$
\overline{\Phi(x)}=T^{-1} \int_{0}^{T} \Phi(x, t) \mathrm{d} t
$$

Phase-averaged properties on the other hand were determined in the context of Hussain's (1986) double decomposition, where $\Phi$ is decomposed into coherent and incoherent components as $\Phi(\boldsymbol{x}, t)=\langle\Phi(\boldsymbol{x}, t)\rangle+\Phi^{\prime}(\boldsymbol{x}, t)$. Here the phase average $\langle\cdot\rangle$ is the ensemble $\mathrm{E}\{\cdot\}$ of all successive structures at the same age or phase $\phi$ in their evolution and $\Phi^{\prime}$ are random fluctuations about that coherence. Thus

$$
\langle\Phi(\boldsymbol{x}, t)\rangle=\mathrm{E}\left\{\left.\Phi(\boldsymbol{x}, t)\right|_{\phi^{\dagger}}\right\}, \quad \text { where } \quad \phi-\frac{\phi_{B}}{2} \leqslant \phi^{\dagger} \leqslant \phi+\frac{\phi_{B}}{2}
$$

and $\phi_{B}$ is the width of the phase bins. Here $\phi_{B}=2 \pi / 16$, since the cycle was broken into sixteen phase bins.

\subsection{Structure identification}

Impinging jets, like other turbulent flows, are dominated by spatially coherent, temporally evolving vortical motions known as coherent structures. Unfortunately the precise identification of these structures remains elusive, although an intuitive method to isolate them is to visualize surfaces of constant vorticity magnitude $|\omega|$. This technique is widely used, but although reliable in free shear layers its use in bounded shear layers is questionable, particularly when the shear and structure are composed of vorticity of similar magnitude.

Techniques that better discriminate between shear and structure are based upon the three invariants $C, Q$ and $R$, and eigenvalues $\sigma$, of the velocity gradient tensor $\nabla \boldsymbol{u}$. Here $\sigma$ satisfies the characteristic equation

$$
\sigma^{3}-C \sigma^{2}+Q \sigma-R=0
$$

where for incompressible flow $C \equiv u_{i, i}=0, Q \equiv-\frac{1}{2} u_{i, j} u_{j, i}$ and $R=\operatorname{Det}\left(u_{i, j}\right)$.

Hunt, Wray \& Moin (1988) defined an eddy as a region of positive second invariant $Q$ of $\nabla \boldsymbol{u}$, while Chong, Perry \& Cantwell (1990) suggested using complex eigenvalues of $\nabla \boldsymbol{u}$ which occur when $(Q / 3)^{3}+(R / 2)^{2}>0$. Jeong \& Hussain (1995) point out limitations with both definitions and introduce their own. Specifically, by noting that 
$Q$ can be written as

$$
Q=\frac{1}{2}\left(\|\Omega\|^{2}-\|\boldsymbol{S}\|^{2}\right),
$$

where $\|\boldsymbol{S}\|=\left[\operatorname{tr}\left(\boldsymbol{S} \boldsymbol{S}^{t}\right)\right]^{1 / 2},\|\Omega\|=\left[\operatorname{tr}\left(\Omega \Omega^{t}\right)\right]^{1 / 2}$, and that $\boldsymbol{S}$ and $\Omega$ are symmetric and antisymmetric components of $\nabla \boldsymbol{u}$, as $S_{i j}=\left(u_{i, j}+u_{j, i}\right) / 2$ and $\Omega_{i j}=\left(u_{i, j}-u_{j, i}\right) / 2$, they introduce the notion that a vortex core is a connected region with two negative eigenvalues of $\boldsymbol{S}^{2}+\Omega^{2}$. Specifically, since $\boldsymbol{S}^{2}+\Omega^{2}$ is symmetric and thus has three real eigenvalues $\lambda_{1} \geqslant \lambda_{2} \geqslant \lambda_{3}$, their definition is equivalent to the requirement that $\lambda_{2}<0$ within the vortex core. This definition is Galilean-invariant and is consistent with the presence of a local pressure minimum within the flow. Although not definitive (Lugt $1996, \S 12.6$ ), the $\lambda_{2}$ definition is a consistent indicator of structure and we use both it (with $\boldsymbol{u}$ replaced by $\langle\boldsymbol{u}\rangle$ ) and vorticity magnitude in the results to follow. In applying the technique we note that four of the nine components of the velocity gradient tensor follow directly in each measuring plane with a fifth component from continuity; the remainder were calculated using information from adjacent planes.

\subsection{Vector plots and critical point theory}

Velocity vector fields of two-dimensional slices through the flow also provide evidence of structure, particularly when coupled with critical point theory (Lighthill 1963). The same theory, when applied on a plane of symmetry, is also useful in characterizing topological features of the flow. Critical points are points in a flow field where the slope of the instantaneous streamlines is indeterminate. Perry \& Fairlie (1974) classify such points, and theorems regarding their numbers and types are given by Hunt et al. (1978).

To utilize such theory we must compute instantaneous streamlines from the velocity vector field at some instant (phase) on the assumption that the instant is frozen in time. Specifically, since

$$
\frac{\mathrm{d} x}{\mathrm{~d} t}=u(x, y) \quad \text { and } \quad \frac{\mathrm{d} y}{\mathrm{~d} t}=v(x, y),
$$

we integrate in time to obtain first $x(t)$ and $y(t)$, and thence $y=f(x)$. This of course necessitates $u(x, y)$ and $v(x, y)$ being known as continuous functions and so approximations to them were obtained by fitting discrete sets of data with the form

$$
u(x, y), v(x, y) \approx \sum_{k=0}^{m} \sum_{l=0}^{n} A_{k l} \phi_{k}\left(x^{\prime}\right) \Phi_{l}\left(y^{\prime}\right) .
$$

Here $\phi_{k}\left(x^{\prime}\right)$ and $\Phi_{l}\left(y^{\prime}\right)$ are $m+1$ and $n+1$ linearly independent orthogonal functions. Accordingly $A_{k l}$ are chosen to ensure the best approximation in a least-squares sense, which necessitates taking an inner product over both $\phi_{k}\left(x^{\prime}\right)$ and $\Phi_{l}\left(y^{\prime}\right)$ (see e.g. Phillips $\& \mathrm{Wu}$ 1994). Since our domain is bounded, appropriate functions for $\phi_{k}\left(x^{\prime}\right)$ and $\Phi_{l}\left(y^{\prime}\right)$ are Legendre polynomials, where $x^{\prime}$ and $y^{\prime}$ are scaled versions of $x$ and $y$ which lie in the range $[-1,1]$. This procedure is robust and enabled the whole field to be fitted by one surface rather than by the series of overlapping tiles required by Perry \& Tan (1984). Various values of $m$ and $n$ were employed, but in general the error was negligible when $m=n=9$. Equations (2.1) were integrated simultaneously using the method of Bulirsch and Stoer (Press et al. 1986).

Like $\lambda_{2}$, the topology of the flow is Galilean-invariant, but the velocity vector field is not, which means that the ensuing patterns are sensitive to the velocity of the observer. Ideally we should be in the reference frame of the structures, which of course presupposes we know their velocity. In the cases studied by Perry \& Tan, 


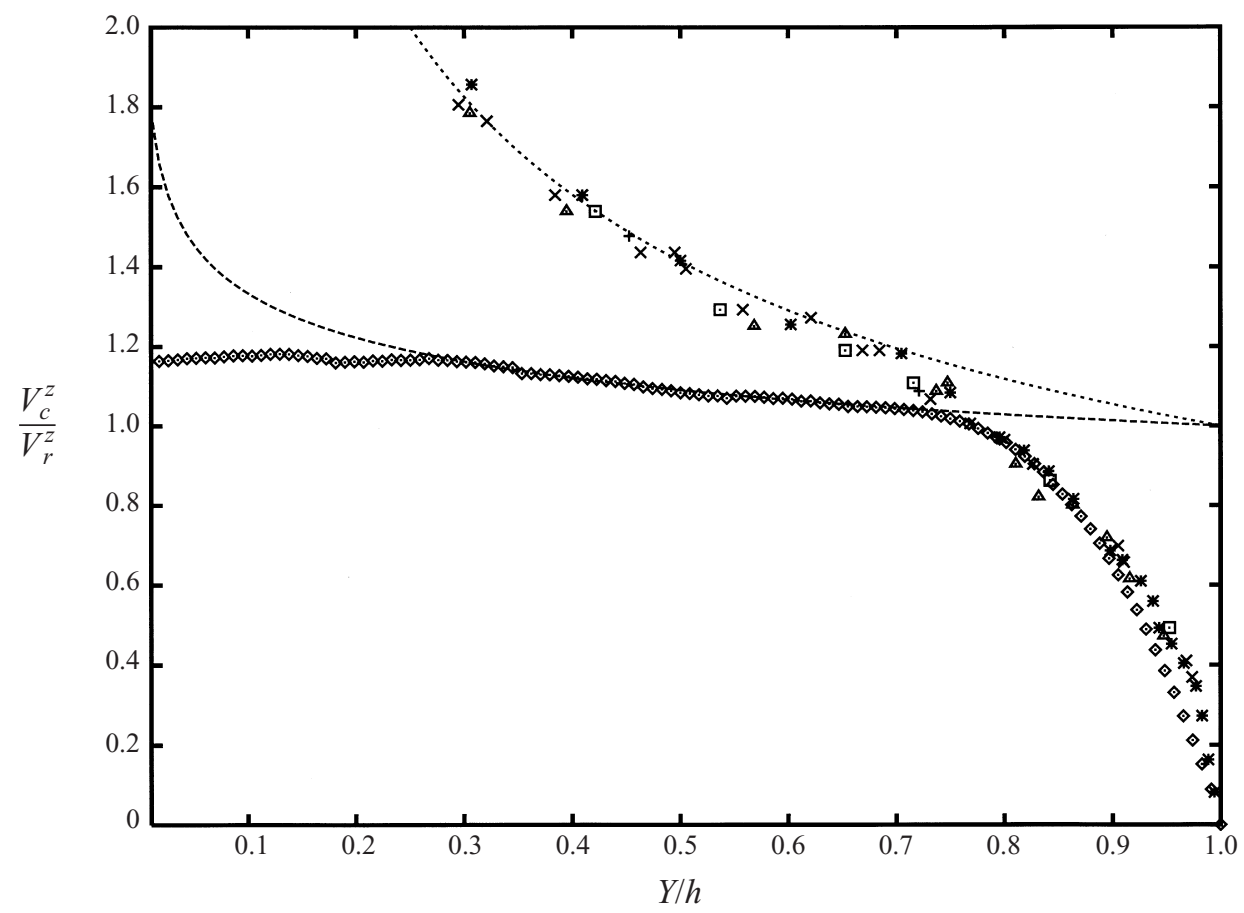

FIGURE 3. Mean velocity distributions on the jet centreline: $\diamond, h=8$, present measurements averaged over $z, R e=2 \times 10^{3} ;+, \square, \times, \triangle, h=14,31,43.6,67.5$, Beltaos \& Rajaratnam (1973), $R e=5.6 \times 10^{3}$; $*, h=100$, Gutmark et al. (1978), Re $=3 \times 10^{4}$; curves: free jet $m=1 / 2$; forced jet $m=1 / 8$.

that velocity was essentially constant and could be deduced from their data, but that is not the case here. To proceed, therefore, we employ a technique from secondary instability theory (see Herbert 1988; Phillips, Wu \& Lumley 1996) which takes the view that structures are three-dimensional perturbations about a mean structureless flow, which in our case is given by $(U, V, W)$ averaged in $z$, i.e. $\left(U^{z}, V^{z}, 0\right)$. Thus $\boldsymbol{u}$ in (2.1) and (2.2) is replaced by $\left(\langle u\rangle-U^{z},\langle v\rangle-V^{z},\langle w\rangle\right)$.

\section{Mean flow characteristics}

We begin our discussion with the time-mean flow field, henceforth called the mean flow field, and in particular by considering the velocity distribution along the centreline of the jet. Here, because of our spatial locking, and in order to compare our results with those of others, we take the $z$-average of $V(0, Y, z)$, i.e. $V_{c}^{z}(Y)$, figure 3 . As evident in the figure, our flow field is composed of three distinct regions namely exiting the jet $(\S \S 3.2,4.1)$, realizing an asymptotic state $(\S 4.2)$ and impinging on the plate $(\S \S 3.3,4.3)$, and we shall consider each separately.

\subsection{The time-mean velocity field}

Because the centreline velocity of unbounded jets decays, say, as $\left(Y / h+Y_{0} / h\right)^{-m}$ ( $m$ constant), where $Y_{0}$ is a virtual origin, we refer $V_{c}^{z}$ to a reference velocity $V_{r}^{z}=$ $\left[\left(Y_{r}+Y_{0}\right) /\left(h+Y_{0}\right)\right]^{m} V_{c}^{z}\left(Y_{r}\right)$ at some value $Y=Y_{r}$ along the jet. Previous works (Beltaos \& Rajaratnam 1973; Gutmark et al. 1978) argue that $Y_{0} / h \ll 1$ and thus ignore $Y_{0}$. They then set $Y_{r}=1$; but since the jet takes time to relax to its asymptotic state, we prefer $Y_{r}=h / 2$. Other sets of data are also shown in figure 3. These studies 


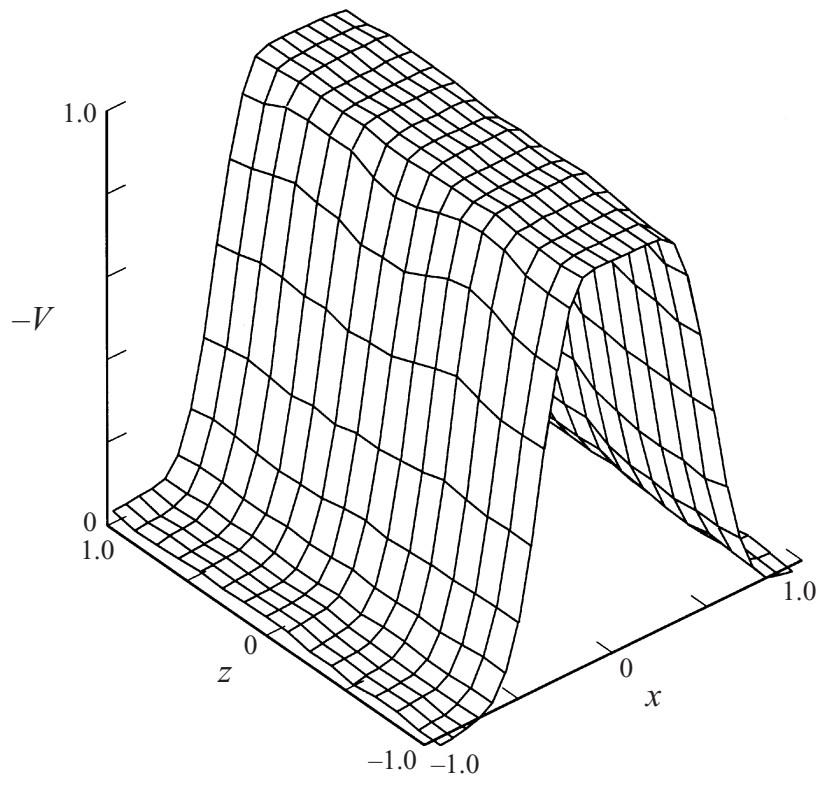

FIGURE 4. Mean velocity distribution of the jet at the streamwise location $Y=1$.

employed free jets $(m \approx 1 / 2)$ with $h$ varying from 10 to 100 , while in the present work the jet was forced, yielding $m \approx 1 / 8$. Observe that in spite of these differences, and differences in Reynolds number, the data in the impingement region agree well. Indeed in all instances the impingement region is seen to occupy about a quarter of the distance between the plate and nozzle exit. Finally Hiemenz flow requires the velocity to asymptote to a linear increase with distance from the plate (see Batchelor $1967, \S 5.5)$ and the data conform.

\subsection{Near the nozzle exit}

The mean velocity $V$ at a streamwise location of $Y=1$ is shown in figure 4 . Observe that $V$ is essentially uniform except in the shear layer resulting from the nozzle boundary layer. The momentum thickness of the boundary layer at the nozzle exit was about $0.028 b^{*}$, but slight variations about that thickness owing to the fluted nozzle edge, and with the same wavelength as the flutes, are evident in the shear layer downstream. These variations are not evident in the jet core, although it was subject, in the absence (presence) of artificial excitation, to fluctuations with a streamwise intensity of $0.007 V_{0}^{*}\left(0.009 V_{0}^{*}\right)$.

\subsection{Near the plate}

The mean velocity field in the vicinity of the plate is shown in figures $5(a)$ and $5(b)$. Figure $5(a)$ depicts mean velocity vectors $i U+\boldsymbol{j} V$ in the $(x, y)$-plane through $z=0$. Observe that jet divergence due to the plate can be traced (in accord with figure 3) to at least $y=2$ and that the boundary layer assumption $\partial U / \partial x \ll \partial U / \partial y$ would appear to be satisfied for $|x|>2$. The region bounded (loosely) by these $x$ and $y$ values is usually termed the stagnation region, owing to the presence of a stagnation point or line on $y=0$. However, except at random points in time, the flow therein is anything but stagnant and the term therefore misleading; for that reason we refer to this region as the 'impingement region'. Overlaid on the mean velocity field are contours of mean spanwise vorticity $\bar{\omega}_{z}$. Observe that vorticity generated in the 
(a)

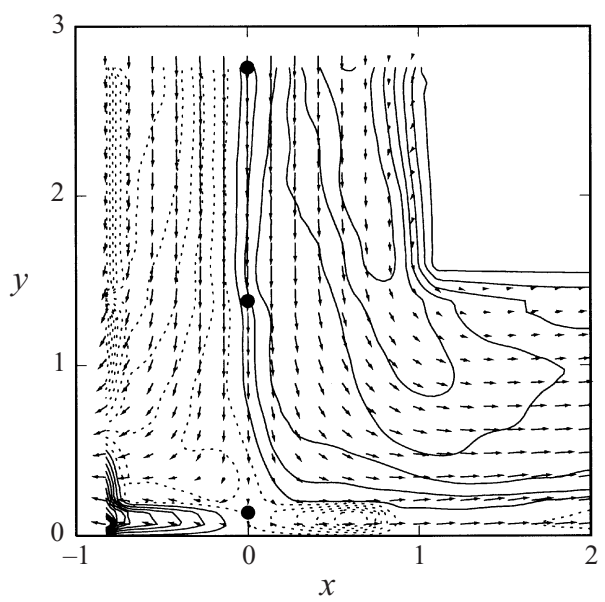

(b)
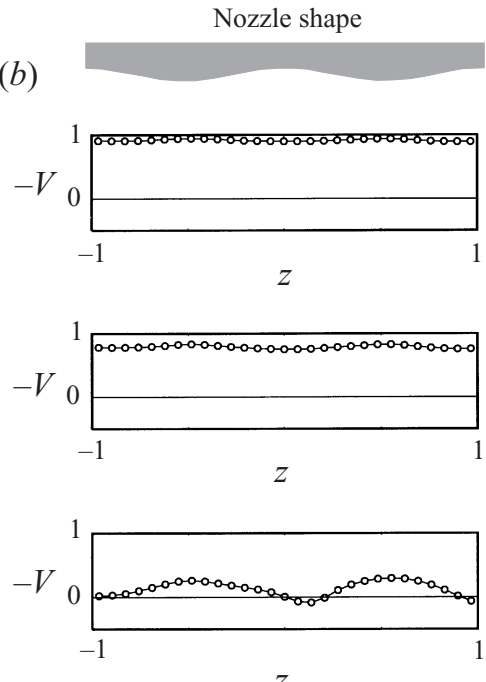

FIGURE 5. The impingement region showing: $(a)$ mean velocity vectors $i U+\boldsymbol{j} V$ overlaid with contours of mean spanwise vorticity $\bar{\omega}_{z}$ in the $(x, y)$-plane at $x=0 ;(b)$ spanwise variations of mean velocity on $x=0$ at various $y$ locations indicated by $\bullet$ in $(a)$.

stagnation-point boundary layer is confined to $y<0.3$. This vorticity is of opposite sign to that from the jet and suggests that the bulk of the impingement region is infused with vorticity from the jet rather than from the boundary layer. Thus while the influence of the wall plays a crucial role in the large-scale vorticity dynamics in the impingement region, the role of wall-generated vorticity $\bar{\omega}_{z}$ is probably minor for those dynamics.

Figure 5(b) shows spanwise perturbations of the mean velocity $V$ at three $y$ locations in the $(y, z)$-plane at $x=0$; for orientation the $y$ locations are marked with a bullet in figure $5(a)$, and the curves in figure $5(b)$ are aligned with these points. Observe that the perturbations have the same wavelength as, but are of opposite phase to, the fluted nozzle. These perturbations set the spanwise scale of structure throughout the flow and we now turn attention to that structure.

\section{Phase-averaged structures}

As noted in $\S 3$, the flow field is composed of three distinct regions and we shall consider each in order. Flow structure is indicated by iso-vorticity surfaces in regions away from the wall and iso-vorticity surfaces supplemented by other techniques close to the wall.

\subsection{Near the nozzle exit}

Figure 6 depicts surfaces of iso- $\left|\left\langle\omega_{z}\right\rangle\right|$ at $Y=1$. Observe that the rollers occur symmetrically with respect to the centreplane of the jet and depict sinusoidal-like distortions with the same wavelength $\Lambda$ as the flutes, which are drawn for reference at the bottom of the figure. Such features concur with the findings of LC and Rogers $\&$ Moser $(1992 a)$ in plane mixing layers.

Figure 7 shows $x, y$ cross-cuts at $z=0,0.25$ and 0.5 (marked A, B and C) through the iso-surfaces in figure 6, corresponding to a valley, mean and peak of the nozzle flutes. Observe that the rollers are ellipsoidal rather than circular and that the major 


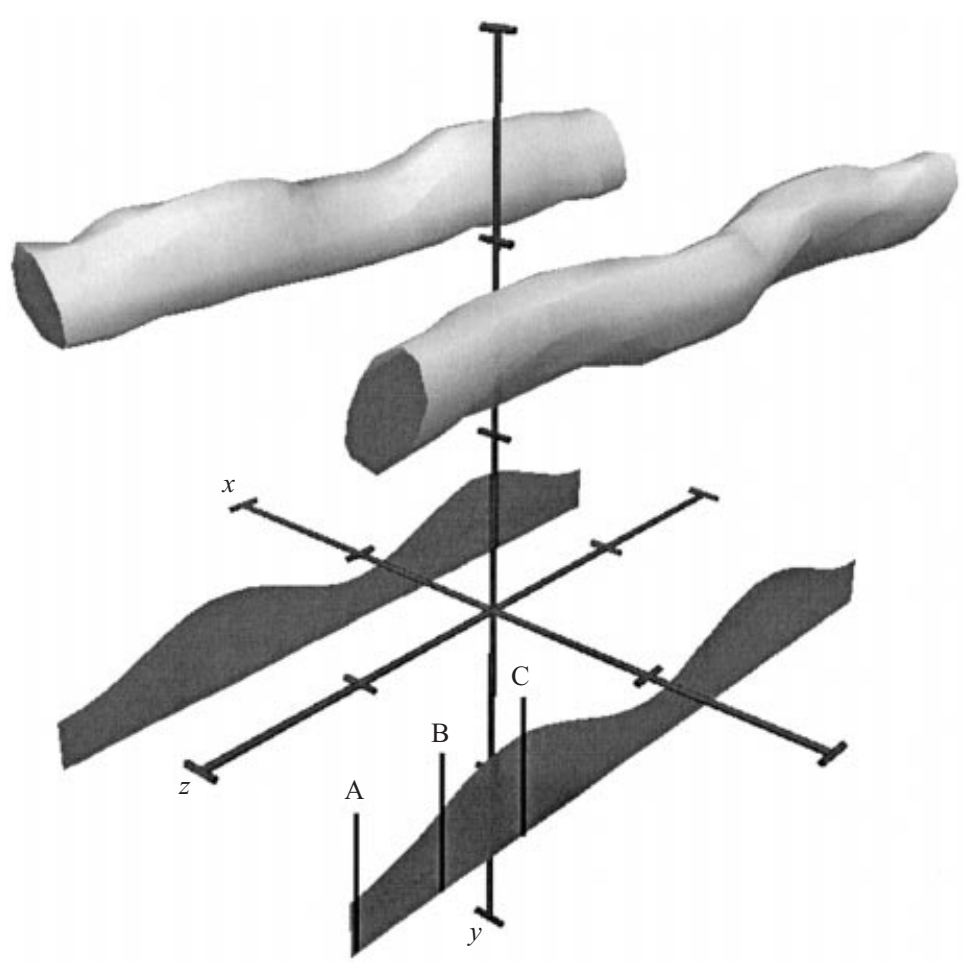

FIGURE 6. Iso-vorticity surfaces of the absolute value of the phase-averaged spanwise vorticity component $\left|\left\langle\omega_{z}\right\rangle\right|=7$, at $Y=1$ and phase angle $\phi=0$. A, B and $\mathrm{C}$ refer to cross cuts shown in figure 7.

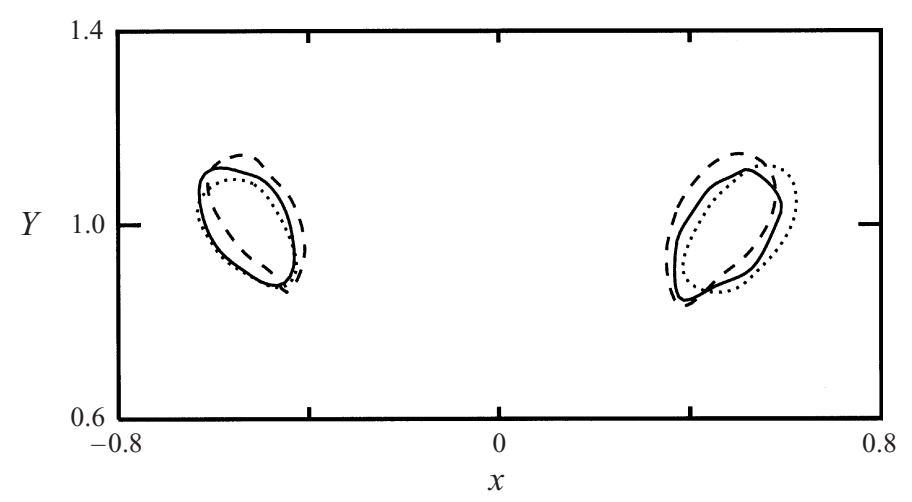

FIgURE 7. Cross-section of spanwise rollers in the planes through A, B and C shown in figure 6; A dotted; B solid; C dashed.

(and minor) axis of the ellipse lies in a plane inclined at roughly $45^{\circ}$ to the vertical. Moreover, relative to the roller core at $z=0.25$, the core at $z=0.5$ is shifted inward from the jet axis and leads in the streamwise $Y$-direction. This means the undulations of the roller are in-phase with the flutes, in contrast with those of LC in a plane mixing layer, whose rollers are phase shifted by $\pi$ with respect to indentations in the splitter plate. In short, the present rollers appear much like an isolated pair of vortex filaments subject to the symmetric long-wave Crow (1970) instability about their minor axis. 

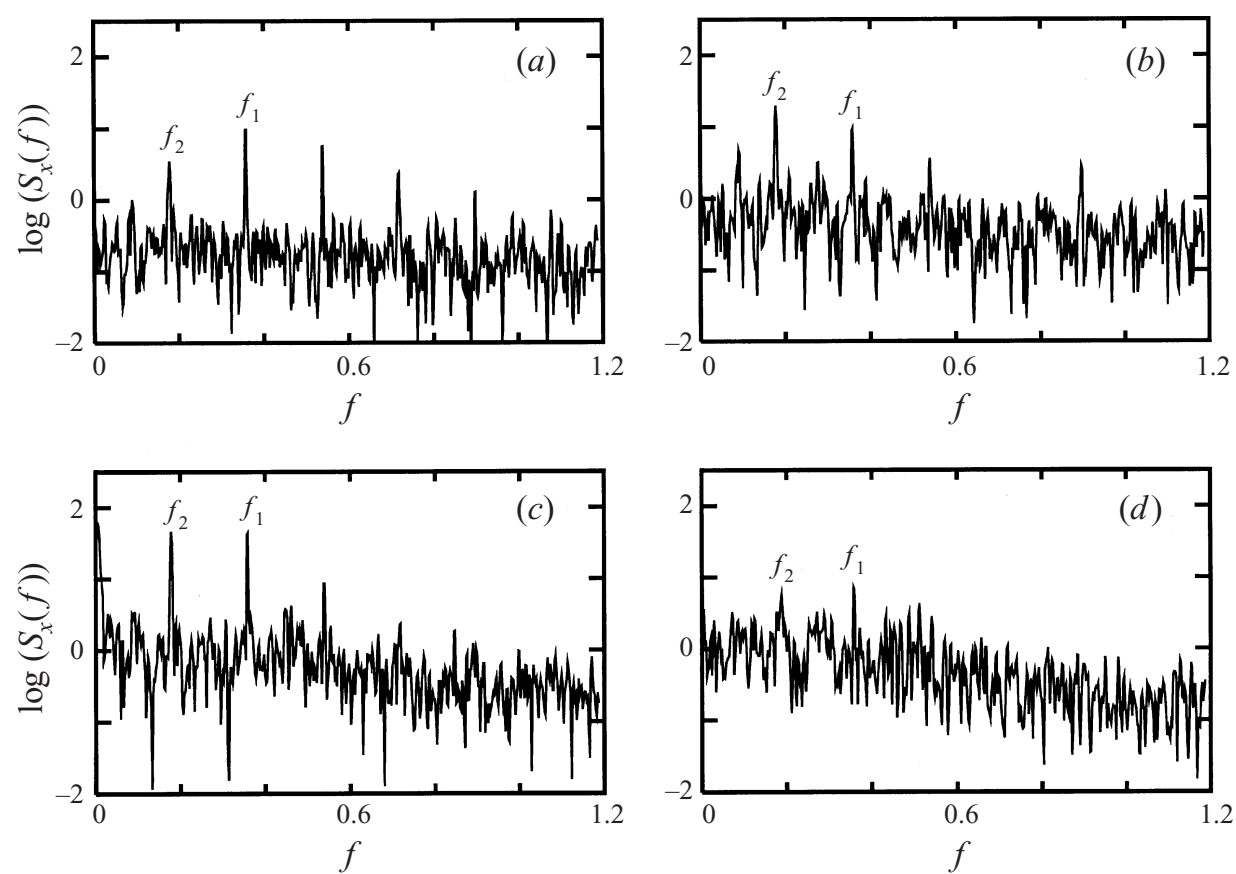

FIGURE 8 . Enstrophy density spectrum of the $\omega_{x}$ component of vorticity measured at $(y, z)=(1.0,0.3)$ with $(a),(b),(c),(d)$ at $x=0,0.5,1.0,1.5$ respectively.

\subsection{Between the nozzle and the impingement region}

According to linear stability analysis, plane mixing layers are unstable to virtually any spanwise wavelength, although the most amplified wavelength is about $2 / 3$ of the spacing between consecutive rollers (Pierrehumbert \& Widnall 1982), and experiments concur (LC; Nygaard \& Glezer 1991). Here the roller spacing is $V_{N}^{*} / f_{1}^{*}$ or approximately $1.45 b^{*}$ since the convection velocity of the rollers (as determined by tracking the peak value of phase-averaged vorticity) is $V_{N}^{*}=0.52 V_{0}^{*}$, so that the ratio $\Lambda^{*} f_{1}^{*} / V_{N}^{*} \approx 0.71$. This ratio has not previously been measured in a plane jet, but it compares favourably with linear stability theory and falls within the 0.5 to 0.8 range observed in plane mixing layers (Leboeuf \& Mehta 1996).

The rollers undergo their only pairing in the vicinity of $y=4$. Huang \& Ho (1990) suggest that pairing in mixing layers is synonymous with a doubling in wavelength of the roller's undulations spanwise (see also Leboeuf \& Mehta 1995, 1996), but exceptions are evident in other experiments (Lasheras, Cho \& Maxworthy 1986; Bell \& Mehta 1992) and direct numerical simulations provide no evidence for it at all (Rogers \& Moser 1992b). Here there was no change in wavelength, but there was a change in the dominant frequency of the flow. That change is evident in figure 8 , where we plot the enstrophy density spectrum $S_{x}(f)$, defined as $\overline{\omega_{x}^{\prime 2}}=\frac{1}{2} \int_{-\infty}^{\infty} S_{x}(f) \mathrm{d} f$ at several points across the shear layer. Observe that the dominant frequency is $f_{2}$, which is half the fundamental frequency, in all cases except at $x=0$. In consequence the appropriate period for phase-averaging in the impingement region is $1 / f_{2}^{*}$, and that was used. $S_{x}(f)$ was measured using PIV data in time series at 20 per second; the maximum measurable frequency was therefore $10 \mathrm{~Hz}$. 

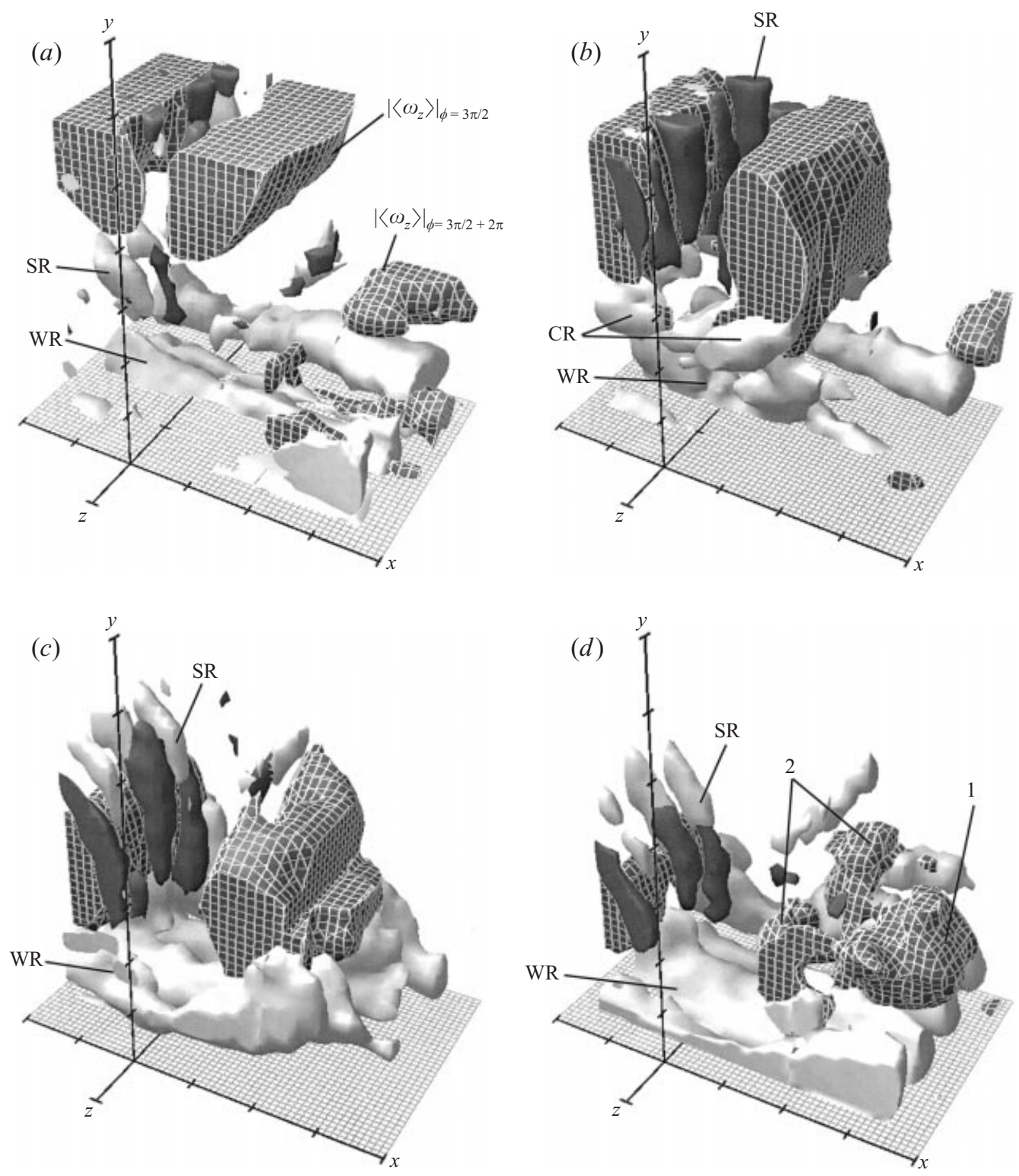

FIGURE 9. Iso-vorticity surfaces near the plate at various phase angles. Light grey surfaces represent $\left|\left\langle\omega_{x}\right\rangle\right|=1.1$; dark grey surfaces represent $\left|\left\langle\omega_{y}\right\rangle\right|=1.1$; cross-hatched surfaces represent $\left|\left\langle\omega_{z}\right\rangle\right|=1.1$. The plate is depicted by a flat mesh with a finer grid. Tic marks are at 0.5 intervals. Cross ribs are denoted CR; successive ribs SR; wall ribs WR. (a) $\phi=3 \pi / 2 ;(b) \phi=0 ;(c) \phi=\pi / 2 ;(d) \phi=\pi$.

\subsection{In the impingement region}

An overview of the structure in the impingement region is given in figure 9 using isovorticity, with a schematic of the structure in figure 10; figure 11 on the other hand gives a side by side comparison of iso-vorticity, $\lambda_{2}$ and instantaneous streamlines. Here we discuss figure 9 where, for orientation, we note that the centreplane of the jet coincides with the $(y, z)$-plane at $x=0$ and that tic marks have been placed at 


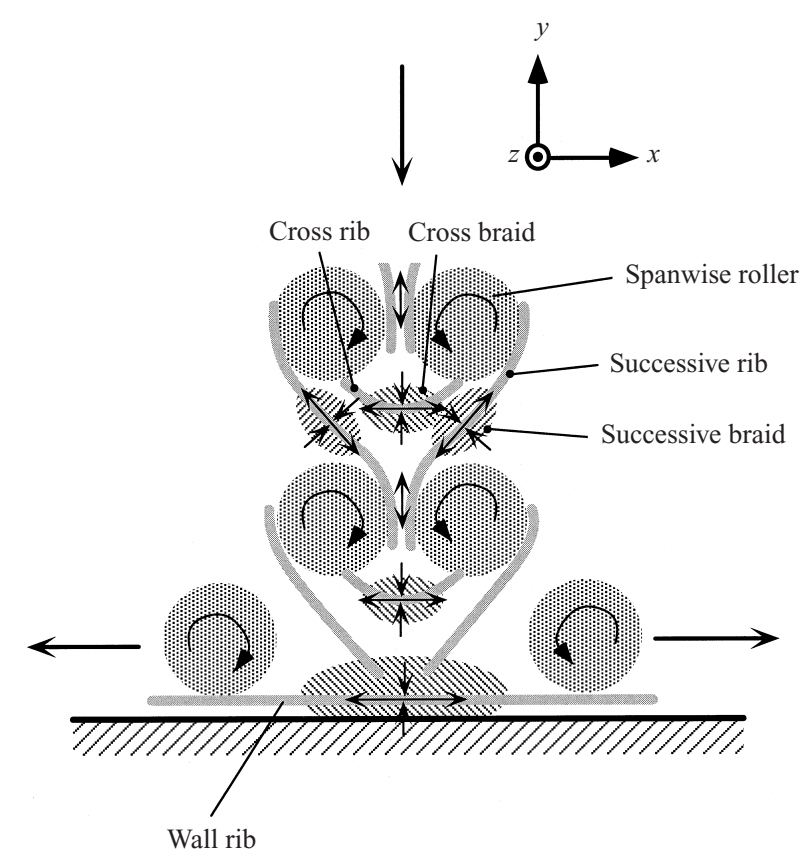

FigURE 10. Schematic of the vortical structure in the impingement region.

0.5 -intervals to provide a depth of scale. Finally, to clarify events near the wall, a thin layer of concentrated spanwise $\left\langle\omega_{z}\right\rangle$ boundary layer vorticity has been removed.

The dominant vortical structures entering the impingement region are a pair of rollers; these are depicted by surfaces of $\left|\left\langle\omega_{z}\right\rangle\right|$. The rollers first convect towards the wall (figure $9 a, b$ ), and then change direction to follow the diverging mean flow (figure $9 c, d$ ). Finally as a new pair of rollers enter the impingement region $\left(\left|\left\langle\omega_{z}\right\rangle\right|_{\phi=3 \pi / 2}\right.$ in figure 9a) the old set exit it $\left(\left|\left\langle\omega_{z}\right\rangle\right|_{\phi=3 \pi / 2+2 \pi}\right.$ in figure $\left.9 a\right)$.

Spanwise periodic perturbations to the rollers are in accord with those observed in the near nozzle region. However the amplitude of the perturbation increases as the roller approaches the wall (figure $9 c$ ) and ultimately contorts into two connected lobes, the frontal one being larger; these are labelled (1) and (2) in figure $9(d)$. The spanwise spacing of each lobe remains about $\Lambda$.

Successive rollers in mixing layers are connected by rib vortices (and braids) (Hussain 1983, 1986; Bernal \& Roshko 1986; Rogers \& Moser 1992a) and that too is the case here, where they are evident as $\left|\left\langle\omega_{x}\right\rangle\right|$ and $\left|\left\langle\omega_{y}\right\rangle\right|$ iso-surfaces in figure $9(c)$, labelled SR. For reasons apparent shortly, we refer to such ribs as 'successive ribs'. Successive ribs extend from the outside portion of the upstream roller to the inside portion of the downstream roller. Moreover, as we shall determine in $\S 5.1$, they retain their orientation as the rollers progress through the impingement region and are strain-dominated and stretched in the direction parallel to themselves, as shown schematically in figure 10. Finally, successive ribs are spanwise periodic with a wavelength of about $\Lambda$.

A spanwise array of similarly spaced rib vortices composed of $\left|\left\langle\omega_{x}\right\rangle\right|$ are evident near the wall. These are quasi-streamwise (in the $x$-direction) and extend through the symmetry plane, as was previously reported by Yokobori et al. (1997), who observed them using the hydrogen-bubble technique. We refer to these as 'wall ribs' and label 
(a) $\phi=3 \pi / 2$
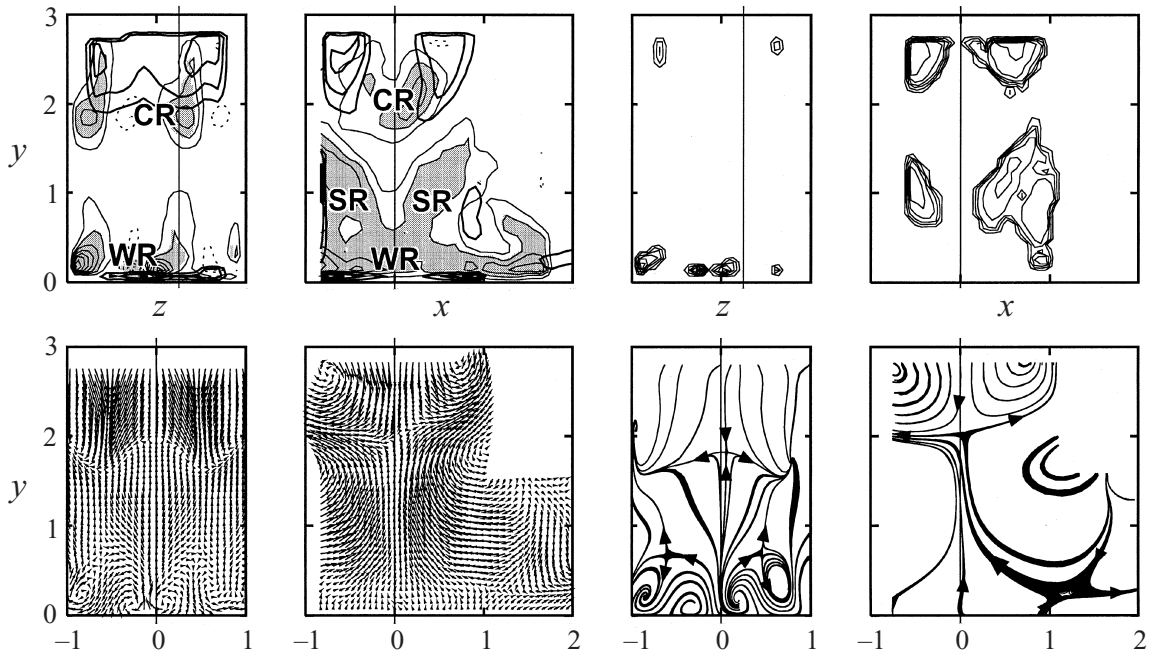

(b) $\phi=7 \pi / 4$
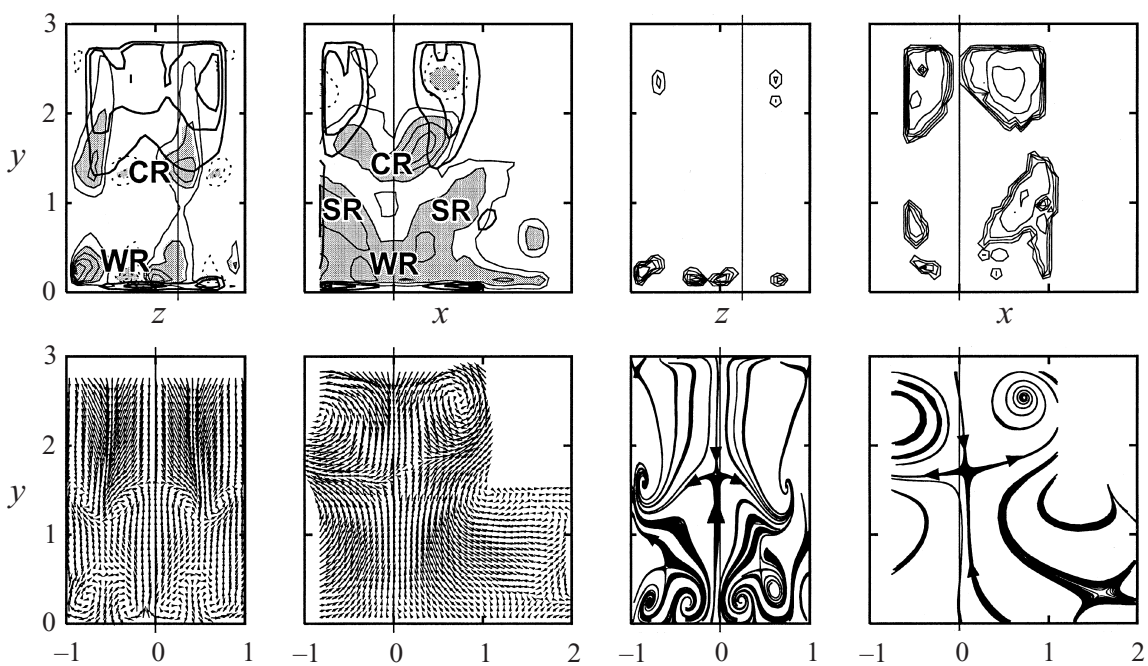

FIGURE $11(a, b)$. For caption see page 291.

them WR. Initially it was thought that wall ribs were the end product of successive ribs, but that in fact is only partly true. Rather, wall ribs are sustained, at least near the symmetry plane, by what we term 'cross ribs'.

Cross ribs extend from the downstream side of each roller to its counterpart across the symmetry plane and are evident as concentrated regions of $\left|\left\langle\omega_{x}\right\rangle\right|$ labelled CR in figure $9(b)$. We shall discuss cross ribs further in $\S \S 5.1,5.2$. Cross ribs are perhaps to be expected in a plane jet with combined fundamental and subharmonic forcing in phase because the rollers are symmetrical rather than staggered, but they have not previously been reported. This may be because their influence in unbounded shear layers is minor, whereas here their importance is emphasized by stretching as they approach the wall. Nevertheless, we must prove they exist and ask how they came to be, and we shall do so by using vector velocity plots, critical point theory and $\lambda_{2}$ in $\S 5$. 
(c) $\phi=0$
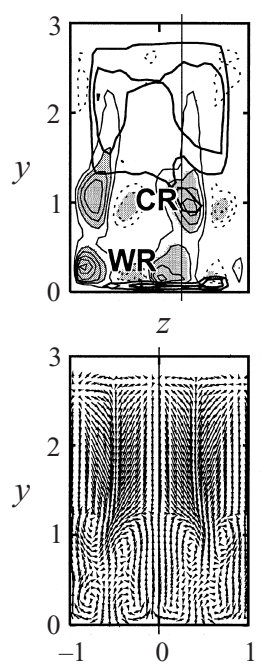

(d) $\phi=\pi / 4$

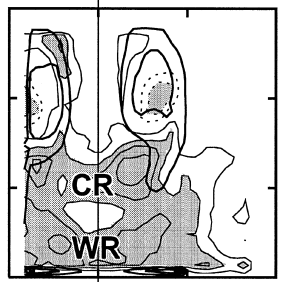

$x$

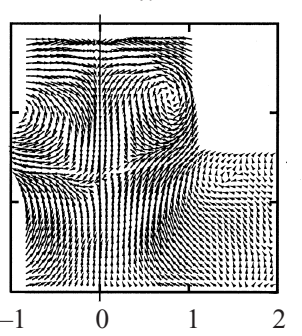

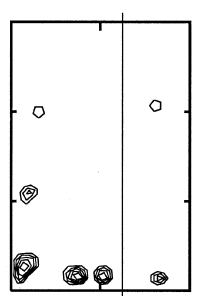

$z$

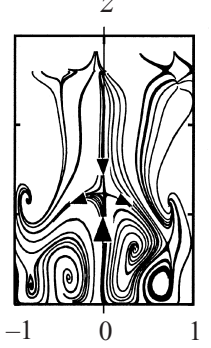

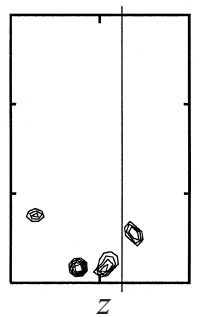

$x$
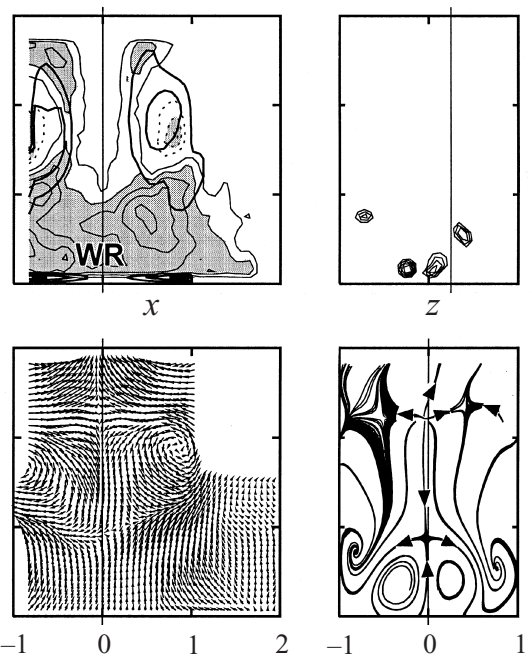

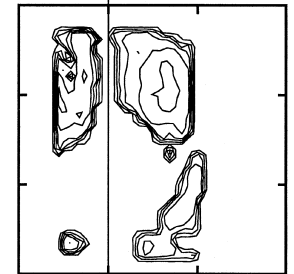

$x$
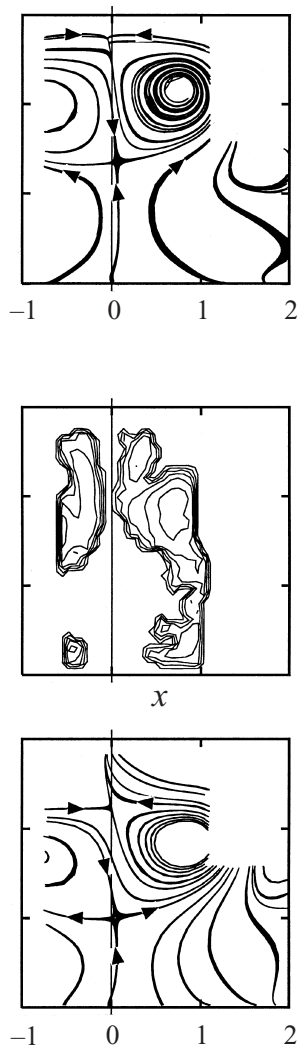

FIGURE $11(c, d)$. For caption see page 291.

\section{Successive, wall and cross ribs}

A schematic of the structure introduced in $\S 4$ is given in figure 10 and we should now like to provide more detail of that structure. Specifically, we begin with evidence of cross and successive ribs and show how each culminate as wall ribs, $\S 5.1$. We then discuss how cross ribs are formed, $\S 5.2$, and go on to determine that successive, cross and wall ribs are strain dominated, $\S 5.3$. Finally we determine that stretching plays a key role in the production of wall rib vorticity, §5.4.

\subsection{Structures}

Plots of the various identification techniques in two orthogonal planes and at phase intervals of $\pi / 4$ beginning with $\phi=3 \pi / 2$, are given in figure 11 . The upper level at each phase shows iso- $\left\langle\omega_{x}\right\rangle$ and iso- $\lambda_{2}$, while beneath them are velocity vectors relative 
(e) $\phi=\pi / 2$
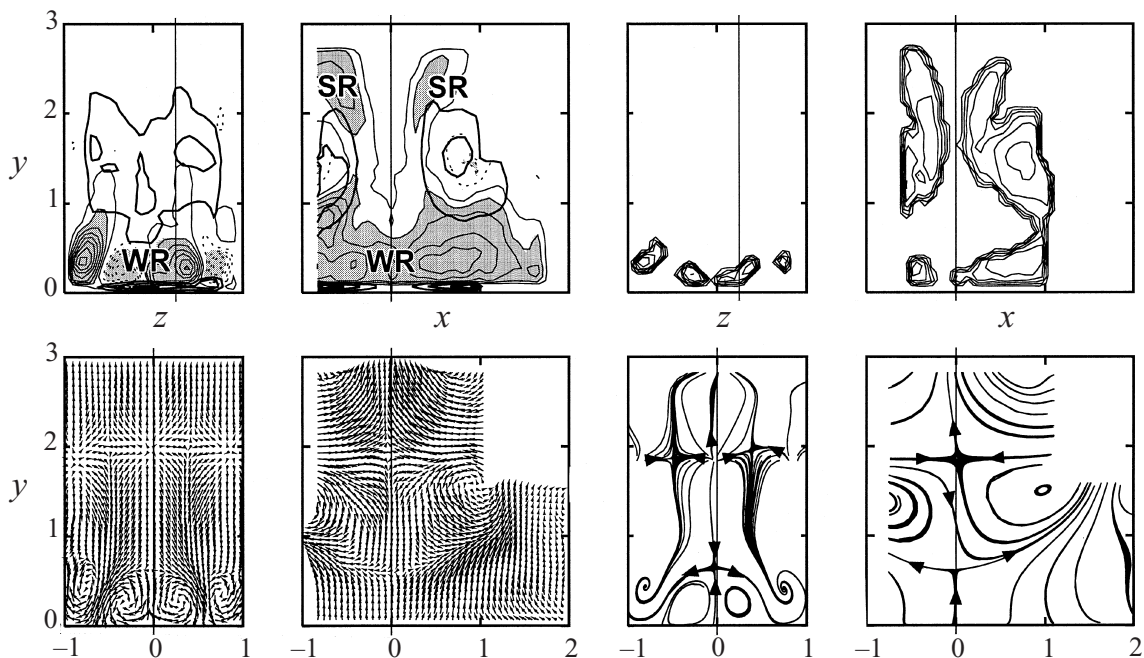

$x$
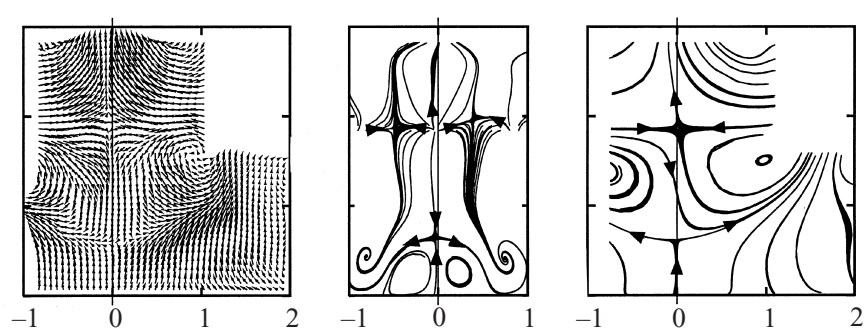

(f) $\phi=3 \pi / 4$
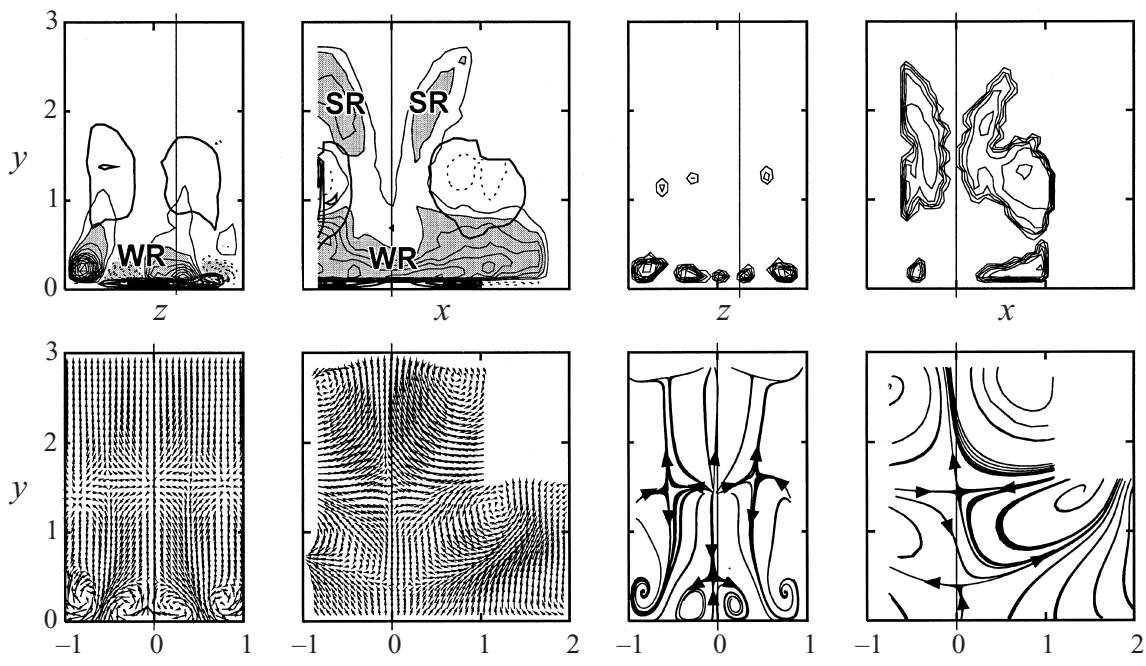

FIGURE $11(e, f)$. For caption see facing page.

to the mean flow as defined in $\S 2.7$ and their instantaneous streamlines. In each instance the left-hand figure is of the $(y, z)$-plane through $x=0$, while the right-hand figure is of the $(x, y)$-plane at $z=0.3$ in the case of $\left\langle\omega_{x}\right\rangle$ and $\lambda_{2}$ (which cuts through successive and wall ribs) and $z=0$ otherwise. A vertical line marks the location of each slice. Furthermore, for orientation the iso-vorticity, henceforth denoted $\Omega$, plots have further features: a shaded region denotes $\left|\left\langle\omega_{x}\right\rangle\right|>0.8$ and thicker contours denote roller position. Finally, because all components of the velocity gradient tensor are required to evaluate $\lambda_{2}$ (see $\S 2.6$ ), we note that its $x$-wise extent is less than that of the other techniques.

We look first at the $x, y$ plots and the rollers. Observe that each technique consistently depicts their presence and position, at least within their respective fields of view. Likewise successive ribs (SR) are clearly evident in the $\Omega$ and $\lambda_{2}$ plots at phases 
(g) $\phi=\pi$
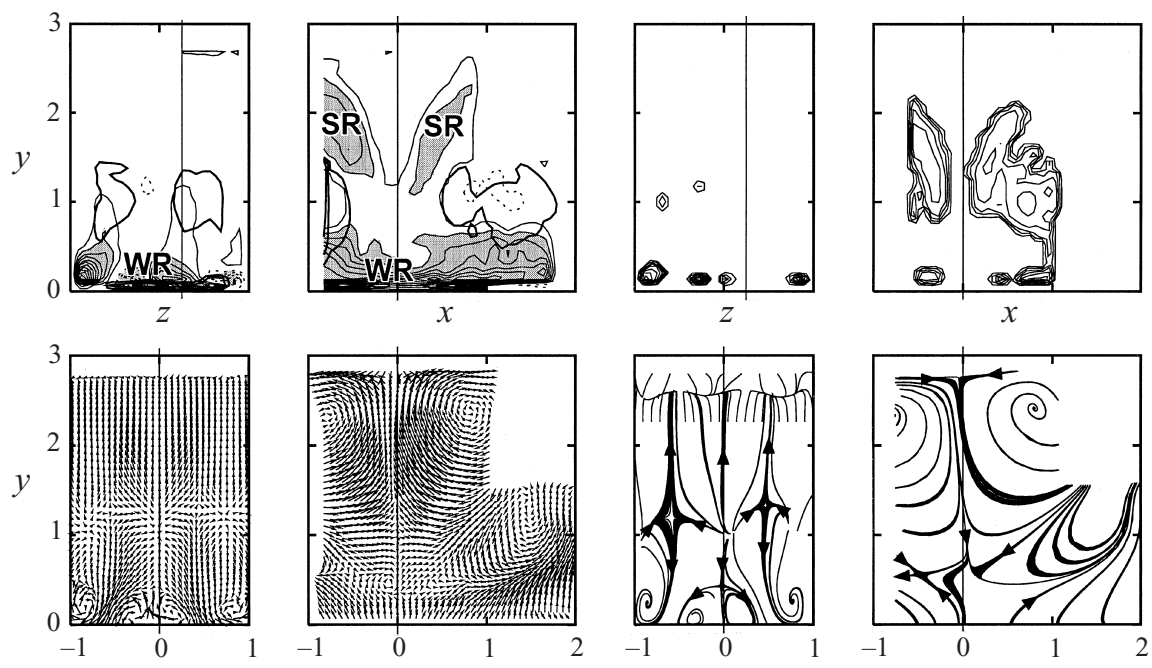

(h) $\phi=5 \pi / 4$
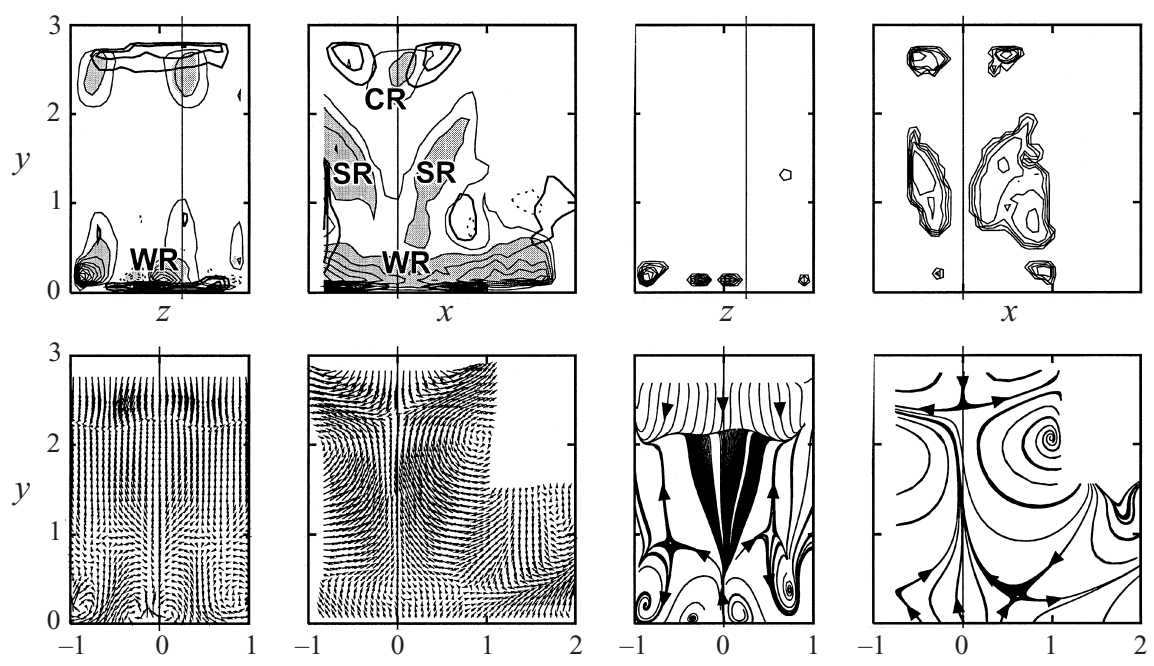

FIGURE 11. Plots of $\left\langle\omega_{x}\right\rangle, \lambda_{2}$, relative velocity vectors and their instantaneous streamlines in two orthogonal planes at phase intervals of $\pi / 4$ beginning at $(a)$ with $\phi=3 \pi / 2$. The left of each figure pair is of the $(y, z)$-plane through $x=0$ while the right-hand side is of the $(x, y)$-plane through $z=0.3$ for $\left\langle\omega_{x}\right\rangle$ and $\lambda_{2}$ and $z=0$ otherwise. A vertical line marks the location of each slice. The shaded region denotes $\left|\left\langle\omega_{x}\right\rangle\right|>0.8$; dotted contours show $\left\langle\omega_{x}\right\rangle$ of opposite sign. Thicker contours are of $\left|\left\langle\omega_{z}\right\rangle\right|$ at $x=0.6$ and $z=0.3$ : these contours indicate roller position and are overlaid for reference. Note also that the field of view of $\lambda_{2}$ in $x$ is restricted to $-0.7 \leqslant x \leqslant 1.03$, while the velocity vectors are relative to the mean flow field as defined in $\S 2.7$.

$(e),(f)$ and $(g)$; and at other phases as well, once we know what to look for. Wall ribs (WR), on the other hand, are present throughout the cycle but come into view (in $z$ ) only at $(e),(f)$ and $(g)$ according to $\lambda_{2}$.

That wall ribs indeed occur throughout the cycle is best seen in the $y, z$ plots, where they are evident with all techniques. Observe that they are spanwise periodic with wavelength $\Lambda$ and that their location is little affected by phase except at $(d)$ where 
they amalgamate with incoming cross ribs (discussed later), which in turn affects their strength, e.g. compare the $\lambda_{2}$ plots at $(a)$ and $(f)$ (see also $§ 5.4$ ).

According to $\Omega$, cross ribs (CR) are first evident at $(h)$ and remain as entities until (c); $\lambda_{2}$ and instantaneous streamlines, henceforth denoted $\psi$, on the other hand do not report them until $(a)$, although $(c)$ again is the last we see of them as entities. But there are noticeable differences from technique to technique, both with regard to the spanwise periodic spacing and $y$-location of cross ribs. Specifically, $\Omega$ implies their spacing concurs with that of wall ribs, namely $\Lambda$, but $\lambda_{2}$ and $\psi$ depict a larger spanwise periodic spacing, indeed $3 \Lambda$ (see e.g. (a)). For an explanation we turn to critical point theory. Here cross ribs are depicted by stable foci (see §5.2) which must, for topological reasons, be separated by a saddle point. It is this saddle point (or more precisely saddle-node trio) that gives, in the vorticity maps, the illusion of a cross rib pair. But $\psi$ and $\Omega$ concur on the $y$-location of cross ribs, placing them closer to the wall than $\lambda_{2}$. Of course $\lambda_{2}$ is Galilean invariant, so its location is indisputable, while the location given by $\psi$ (though not the topology) is affected by the assumed convection velocity; $\Omega$ on the other hand would appear to be influenced by the imposed strain field.

As the cross ribs move toward the wall, the wall ribs beneath them (of the same sign) rise to meet them and the two ultimately merge; compare figures $11(c)$ and $(d)$ in the $\psi$ and $\lambda_{2}$ plots. The merged rib is initially above the wall $\left(\lambda_{2}(d)\right)$, but quickly returns to the wall with enhanced strength. Of course since the spanwise spacing of cross ribs is greater by three than that of the wall ribs, merging occurs with the two outer wall ribs. In short, cross ribs contribute to the survival of the outer wall ribs by periodically supplying them with vorticity of the same sign as each pair of rollers cycle into and through the impingement region. This periodic enhancement of wall rib vorticity acts to increase the cross-sectional area of the ribs $(e)$, while simultaneous stretching (in $x$ ) by the diverging mean flow acts to decrease the cross-sectional area (see $\Omega(f-h)$. The intensity level of wall ribs is thus cyclic (see $\S 5.3$ ).

Successive ribs on the other hand do not penetrate the symmetry plane, as is evident in $(e)$ and $(f)$ and figure 12 , which is a $\psi$-slice parallel to but above the plate. Rather, they remain within the adjacent region $(0.3<|x|<1.0)$ where they appear to retain their orientation until they encounter wall $\operatorname{ribs}\left(\Omega\right.$ and $\lambda_{2}$ figure $\left.11 a-d\right)$. Unlike cross ribs, however, their spacing is close to $\Lambda$, so they probably influence both the inner and outer wall rib pair.

\subsection{Formation of cross ribs}

As the jet shear layers exit the nozzle, they roll up into spanwise rollers (figure 6) separated by a potential core across the plane of symmetry (figure 4). However the extent of the core diminishes as $Y$ increases, until at some point there is a continuous distribution of vorticity (in the mean) across the jet. A continuous distribution of vorticity does not imply cross ribs, however; indeed for cross ribs to form there must be a change in topology and that change is evident in the $y, z \psi$ plots of figure 11 . Cartoons of the two classes of topology depicted in these plots are given in figure 13.

Figure 13(a) is typical of phases $(d-g)$ in figure 11. At the top of this figure is a seperatrix depicting two stable nodes and a saddle point and beneath it another seperatrix depicting two saddle points and an unstable node. Figure $13(b)$, on the other hand, is typical of phases $(h-c)$ and here we find that the stable nodes in the upper seperatrix of figure 13(a) have become stable foci which depict cross ribs. The bifurcation from stable nodes to stable foci is first apparent in figure $11(h)$ and clearly evident in $11(a)$. Accordingly the saddle points in the lower seperatrix of 


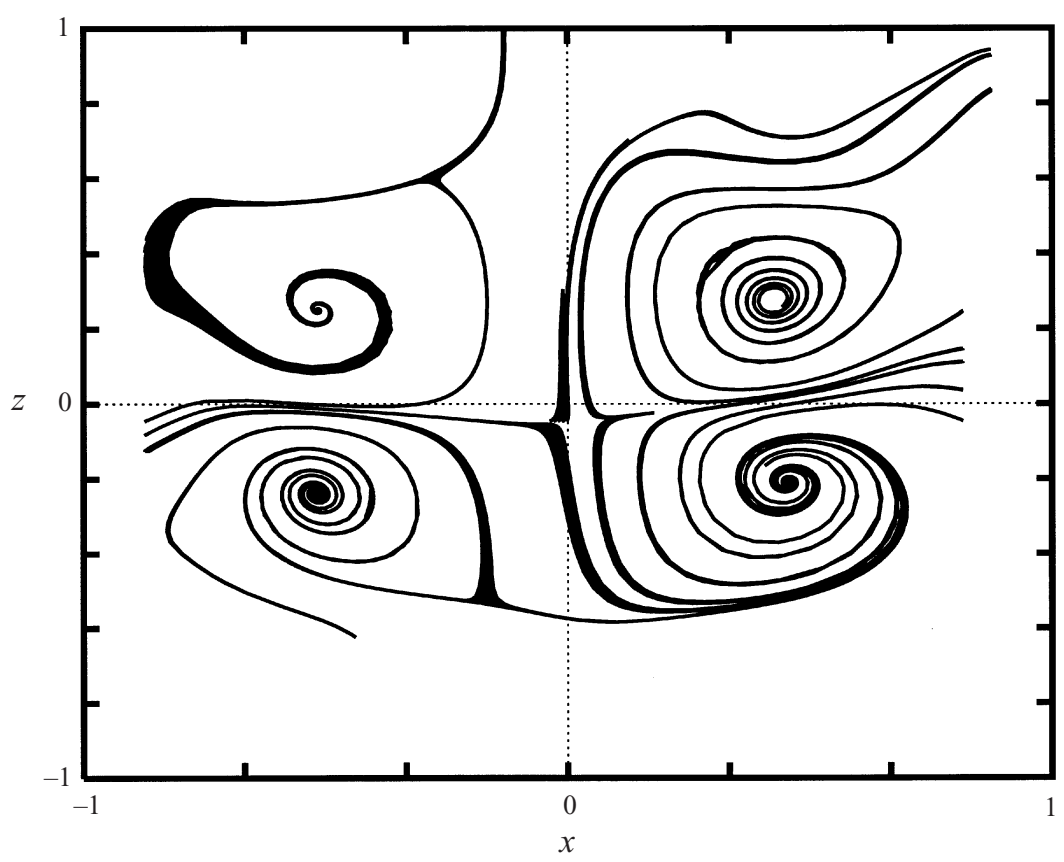

FIGURE 12. Instantaneous streamlines deduced from measured phase-averaged velocity vectors in the $(x, z)$-plane at $y=1.5$. The phase angle corresponds to $(h)$ in figure 11 . The foci depict successive rib vortices that connect spanwise rollers.

figure 13(a) now separate foci depicting wall ribs from those depicting cross ribs. There would also appear to have been a loss of symmetry about the $O-y$ axis at $z=0$.

But why do cross ribs form? As evident in figure 6 and discussed in $\S 4.1$ the rollers undergo a spanwise instability which causes them to distort sinusoidally, much like the Crow (1970) instability. In that instance, which is concerned with an isolated vortex pair, the perturbations are symmetric about the plane of symmetry and the amplitude of the perturbations grows until the vortices touch; they then bifurcate into ring vortices, as seen in figure 116 of Van Dyke's (1982) album of fluid motion. In our case the perturbations also grow until the rollers touch, figure 11(h), but rather than bifurcating to ring vortices, the rollers here stay intact and the bifurcation spawns cross ribs.

The bifurcation is first evident near $y=2.5(Y=5.5)$, just as the rollers enter the impingement region. This, and the fact that the rollers are already unstable at $Y=1$, suggest that the plate is not a factor in the formation of cross ribs, from which we infer that cross ribs may also occur in plane jets. Of course necessary to the formation of cross ribs is that the rollers first undergo a Crow-like instability, but that is not sufficient. For example Rogers \& Moser $(1992 a)$ observe a similar instability in their calculations of a plane mixing layer, but do not observe cross ribs. One obvious difference is that their rollers are antisymmetric about the jet centreline while ours are symmetric, a feature which may well affect the growth rate of the roller perturbation, as may the aspect ratio of the jet.

\subsection{Strain rate tensor}

To ascertain that cross and successive ribs are strain dominated, we turn to the spatial distribution of the principal axes of the phase-averaged symmetric strain- 

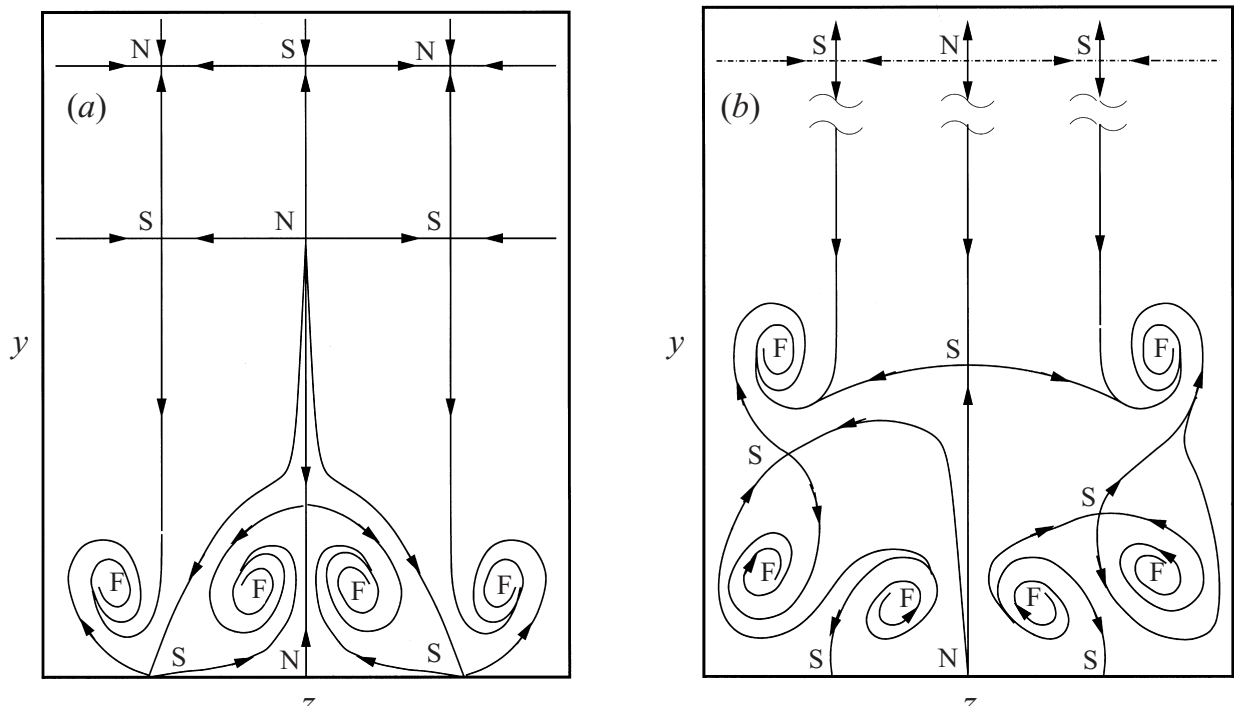

FIGURE 13. Cartoon showing instantaneous streamlines in the $(y, z)$-symmetry plane at $x=0:(a)$ is typical of phases $(d-g)$ in figure 11 , while $(b)$ typifies phases $(h-c)$. The foci $\mathrm{F}$ near the wall in $(a)$ and $(b)$ depict wall ribs while those near the midplane of $(b)$ depict cross ribs. Saddle points are denoted by $\mathrm{S}$ and nodes by $\mathrm{N}$.
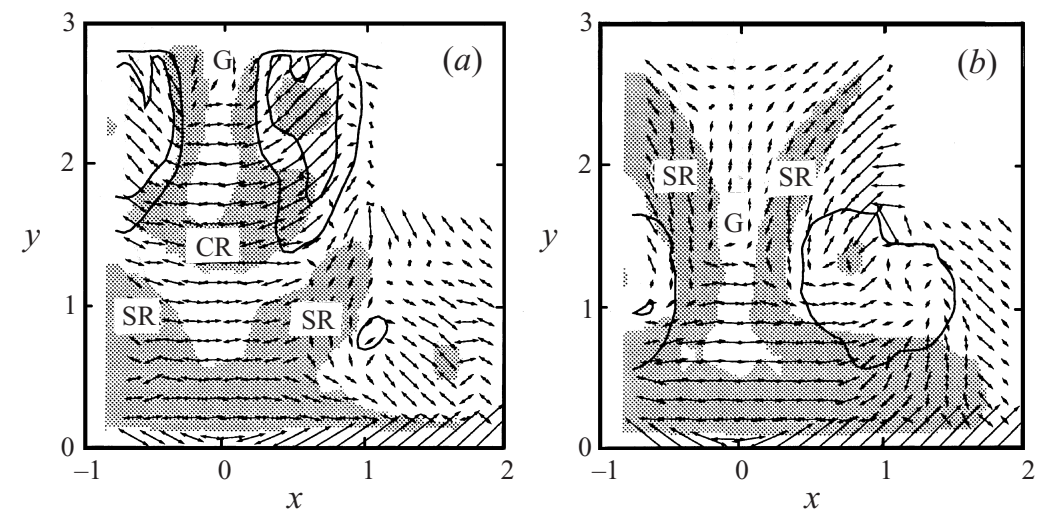

FIGURE 14. Distribution of the direction and magnitude of the expansion of fluid elements as evaluated from the most significant eigenvalue and its vector of the phase-averaged strain-rate tensor. Sections correspond to figures $11(b)$ and $11(f)$ as do reference contours for the rollers; the shaded region depicts $\left|\left\langle\omega_{x}\right\rangle\right|+\left|\left\langle\omega_{y}\right\rangle\right|>0.8$. (a) $\phi=7 \pi / 4$, (b) $\phi=3 \pi / 4$.

rate tensor $\left\langle S_{i j}\right\rangle=\partial\left\langle u_{i}\right\rangle / \partial x_{j}$. This tensor has three unit eigenvectors $e_{i}$, which each denote a principal axis, with corresponding eigenvalues $\gamma$, which denote magnitude, as $e_{i} S_{i j}=\gamma e_{j}$ (Panton 1996, §3.10). Physically, the expansion of a fluid element is dominated by the magnitude and direction of the largest eigenvalue and its vector. The magnitude and direction of the expansion fields corresponding to figures 11(b) and $11(f)$ are shown in figures $14(a)$ and $14(b)$. Here, contrary to figure 11, the shaded region depicts $\left|\left\langle\omega_{x}\right\rangle\right|+\left|\left\langle\omega_{y}\right\rangle\right|>0.8$.

It is evident that cross and successive ribs are aligned with the local direction of expansion, as are wall ribs in the region $|x|<1$. However the converse is not true. If it were we should expect successive ribs to extend to the symmetry plane upstream of the 
rollers, labelled $\mathrm{G}$, where the fluid is expanded in $y$ and compressed in the $x$-direction. But no significant concentrations of $\left\langle\omega_{y}\right\rangle$ or $\left\langle\omega_{x}\right\rangle$ were observed in this region.

\subsection{Production of wall rib vorticity}

Having determined in $\S \S 5.1,5.2$ that wall ribs are periodically fed with vorticity from cross and successive ribs, we should now like to determine whether that enhancement is due to stretching or reorientation of vorticity, or a combination thereof. To do so we consider the dynamical equation for the phase-averaged vorticity (see Hussain 1986), which can be expressed using index notation as

$$
\frac{\mathrm{D}\left\langle\omega_{i}\right\rangle}{\mathrm{D} t}=\left\langle\omega_{j}\right\rangle \frac{\partial\left\langle u_{i}\right\rangle}{\partial x_{j}}+R e^{-1} \frac{\partial^{2}\left\langle\omega_{i}\right\rangle}{\partial x_{k} \partial x_{k}}+\frac{\partial}{\partial x_{j}}\left(\left\langle u_{i}^{\prime} \omega_{j}^{\prime}\right\rangle-\left\langle u_{j}^{\prime} \omega_{i}^{\prime}\right\rangle\right) .
$$

Here the production of vorticity is the first term on the right-hand-side, while viscous diffusion is second and the diffusion by the random fluctuations third. Thus the production of streamwise vorticity $\left\langle\omega_{x}\right\rangle$, can be written in component form as $P_{x x}+$ $P_{x y}+P_{x z}$, where

$$
P_{x x}=\left\langle\omega_{x}\right\rangle \frac{\partial\langle u\rangle}{\partial x}, \quad P_{x y}=\left\langle\omega_{y}\right\rangle \frac{\partial\langle u\rangle}{\partial y} \quad \text { and } \quad P_{x z}=\left\langle\omega_{z}\right\rangle \frac{\partial\langle u\rangle}{\partial z} .
$$

Here $P_{x x}$ is the production of vorticity due to vortex stretching, while $P_{x y}$ and $P_{x z}$ are concerned with reorientation of vorticity due to the local velocity gradient.

Contours of $P_{x x}, P_{x y}$ and $P_{x z}$ in the $(x, y)$-plane are shown in figure 15 at phase angles $(\phi=7 \pi / 4$ and $\phi=3 \pi / 4)$ which correspond to those of figures $11(b)$ and $11(f)$. The $z$-location of the plane also concurs with that of figure 11, as do the shaded region and heavier contours.

At $\phi=7 \pi / 4$ (figure $15 a-c$ ) the rollers have just entered the impingement region. Observe that $P_{x x}$ is significant in both the cross and wall ribs. Conversely, production due to the reorientation of spanwise vorticity $\left(P_{x z}\right)$, and to a lesser extent $\left(P_{x y}\right)$, is evident only in the roller cores (figure $15 b, c$ ), since the sinusoidal distortion of the rollers through $\partial\langle u\rangle / \partial y$ and $\partial\langle u\rangle / \partial z$ redistributes spanwise and normal vorticity into $\left\langle\omega_{x}\right\rangle$. Spanwise rollers in plane mixing layers depict the same phenomena (Rogers \& Moser 1992a).

Half a cycle later $(\phi=3 \pi / 4)$, the production of vorticity due to vortex stretching is even more significant (figure $15 d$ ), while the reorientation of vorticity $\left(P_{x y}\right.$ and $\left.P_{x z}\right)$ is negligible (figure 15e,f). We conclude therefore that rib vortices are sustained by merging and stretching of vorticity, in accord with our observations in $\S 5.2$, not the reorientation of vorticity. In consequence we now turn to the phase evolution of the stretching process.

The stretching term $P_{x x}$ is composed of $\left\langle\omega_{x}\right\rangle$ and $\partial\langle u\rangle / \partial x$, and we are interested in the phase evolution of each. Since local readings of these quantities can be misleading we employ spanwise averages over one wavelength; we then obtain $\left|\left\langle\omega_{x}\right\rangle\right|^{z}$ and $\langle\partial u / \partial x\rangle^{z}$ whose values at $y=0.28$ are plotted in figure 16. Looking first at $\langle\partial u / \partial x\rangle^{z}$ we observe results much as expected in stagnation point flow: specifically, since $\langle u\rangle=0$ on the plane of symmetry and is non-zero for $y>0$ elsewhere, we expect $\langle\partial u / \partial x\rangle^{z}$ to be a maximum on $x=0, y>0$, and that is what the data depict, although details vary throughout the cycle. The cycle for $\left|\left\langle\omega_{x}\right\rangle\right|^{z}$, however, is more varied as we see in figure $16(b)$.

First, in accord with our observation of wall ribs in figure 11, we see that $\left|\left\langle\omega_{x}\right\rangle\right|^{z}$ is non-zero throughout the whole cycle and has a lower bound (dashed line left) in the vicinity of 0.6. Second we note that $\left|\left\langle\omega_{x}\right\rangle\right|^{z}$ at $x=0$ at all times exceeds the lower 

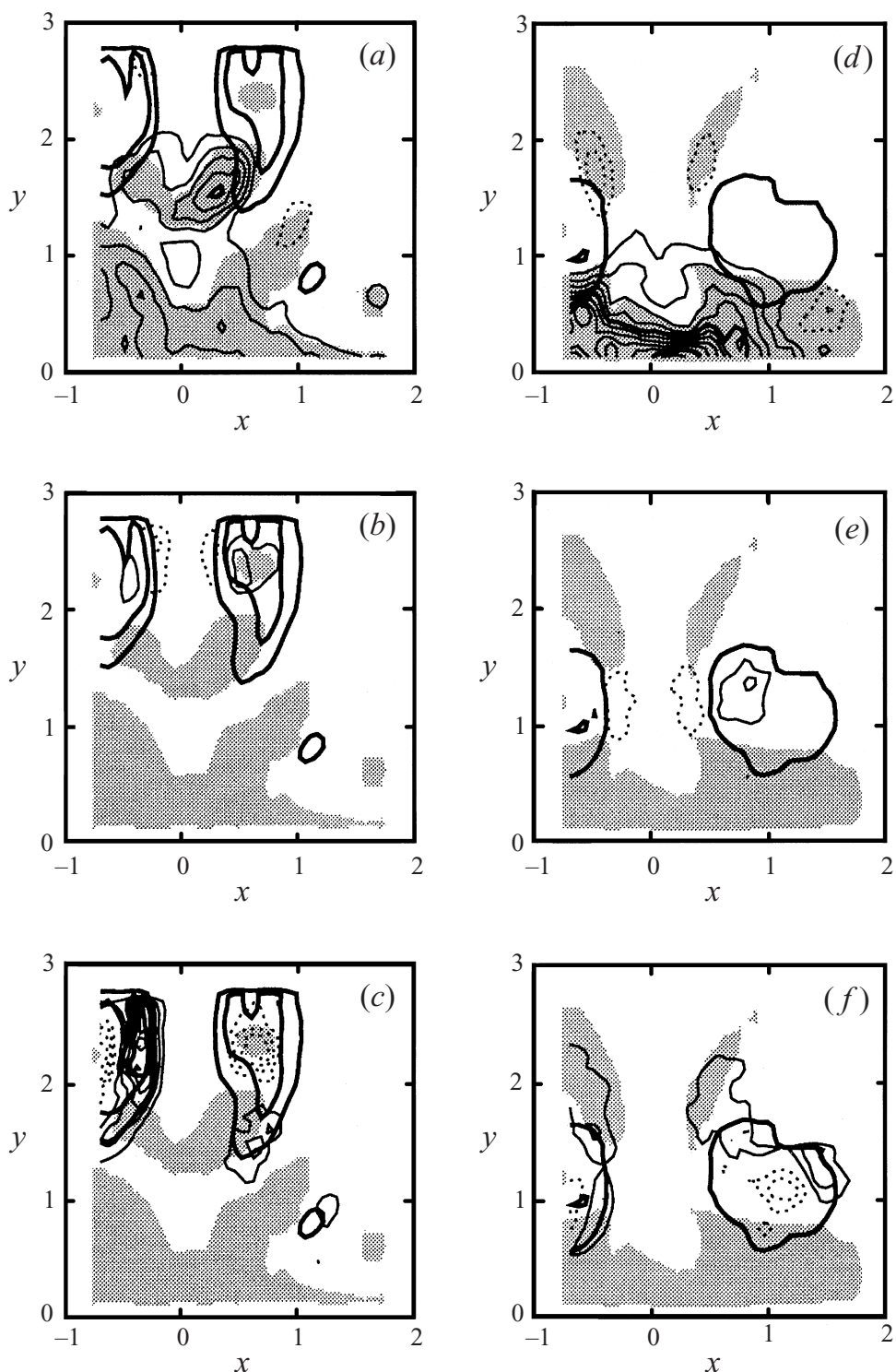

FIGURE 15. Spatial distribution of terms $P_{x x}(a, d), P_{x y}(b, e)$ and $P_{x z}(c, f)$ which contribute to the production of phase-averaged vorticity $\left\langle\omega_{x}\right\rangle$. These are shown in the $(x, y)$-plane at phase angles $\phi=7 \pi / 4(a-c)$ and $\phi=3 \pi / 4(d-f)$. The plane location and phase angles correspond to those of figures $11(b)$ and $11(f)$. Shading and the thicker contours are as in figure 11.

bound, whereas $\left|\left\langle\omega_{x}\right\rangle\right|^{z}$ at other $x$ recover it. We next note that there is an envelope (dashed line right) to which, over varying degrees of $\phi$, all data collapse, suggesting little change in $x$ (at fixed $\phi$ ).

Observe that the $x=0$ curve collapses only near its peak value and exceeds the envelope at all other $\phi$. This supports the notion that vorticity from the cross ribs is for the most part fed to the wall ribs in the vicinity of the symmetry plane, a notion reinforced by the fact that $\left|\left\langle\omega_{x}\right\rangle\right|^{z}$ peaks at $x=0$. This peak occurs at $\phi \approx 3 \pi / 4$ (see also figures $11 f, 14 b, 15 d$ ); that is, when the roller is about midway through 


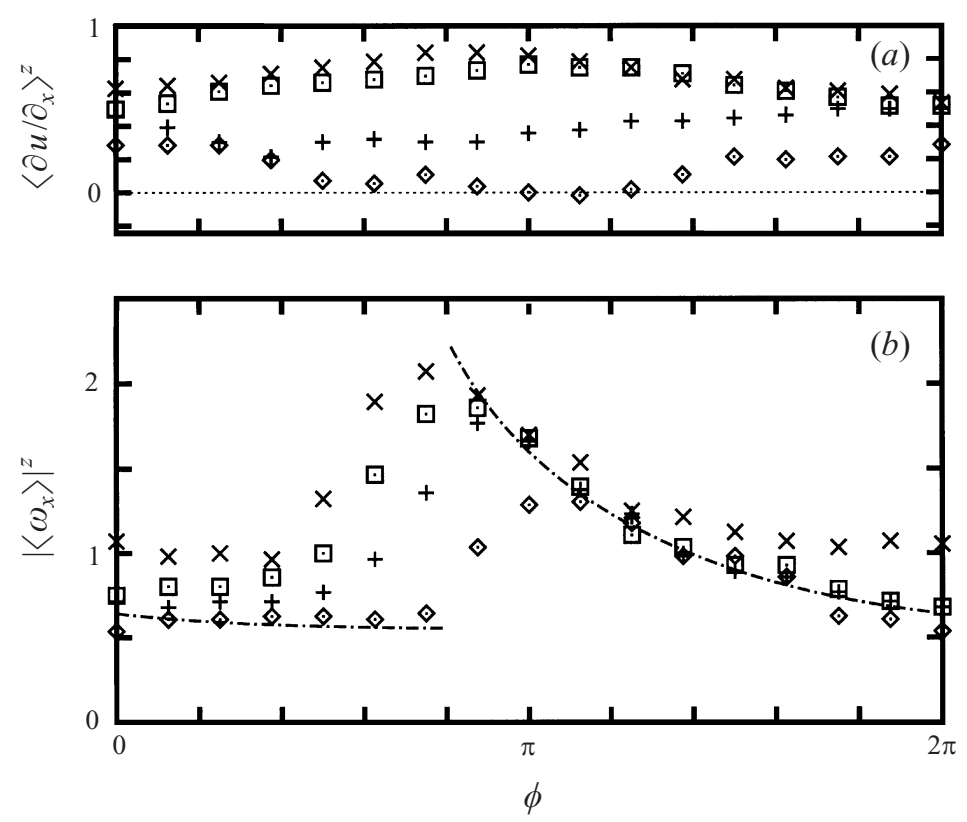

FIGURE 16. Phase distribution of spanwise- and phase-averaged quantities at $y=0.28$ and various $x$-locations: $\times, x=0 ; \square, x=0.5 ;+, x=1 ; \diamond, x=1.5 ;(a)\left|\left\langle\omega_{x}\right\rangle\right|^{z},(b)\langle\partial u / \partial x\rangle^{z}$.

the impingement region. Here the roller acts to enhance strain in the $x$-direction and directly supply $\left\langle\omega_{x}\right\rangle$ to the wall ribs; both effects give rise to the production of $\left\langle\omega_{x}\right\rangle$. Indeed, looking at production, we see that both $\left|\left\langle\omega_{x}\right\rangle\right|^{z}$ and $\langle\partial u / \partial x\rangle^{z}$ are in phase at $x=0$ but that they shift in phase as $x$ increases. In consequence the level of production is highest at $x=0$ and, since $\langle\partial u / \partial x\rangle^{z}$ diminishes to near zero by $x=1.5$, is effectively zero there. However, in spite of the continual production of vorticity at $x=0$ and elsewhere, its absolute level diminishes noticeably with $\phi$. This is most likely caused by viscous and turbulent diffusion.

\section{Summary and discussion}

The DPIV technique employed in the phase-locked experiment described allowed the measurement of entire velocity vector fields of the periodic flow. It also allowed, by use of iso-vorticity, critical point theory and $\lambda_{2}$, a glimpse of the dominant periodic eddying motions that play a role in such flows, both near the jet nozzle and in the impingment region of the plate. Away from the plate the flow was seen to behave in much the same manner as that of a mixing layer, in that the shear layers roll up into spanwise rollers. However while the rollers in a mixing layer occur alternately about its midplane, they here occur symmetrically. Furthermore, in accord with mixing layers (Rogers \& Moser 1992a), the rollers develop a spanwise perturbation with the same wavelength as the fluted jet nozzle and evolve much as do a pair of isolated vortex filaments subject to the symmetric long-wave Crow (1970) instability.

Also in accord with previous observations (Hussain 1983, 1986; Bernal \& Roshko 1986; Rogers \& Moser 1992a) the rollers were connected by ribs. These are spanwise periodic with the spacing of the flutes of the jet nozzle. This spacing was determined to be about 0.71 of the spacing between successive rollers, a value that compares well both with linear stability theory (Pierrehumbert \& Widnall 1982) and observation in 
mixing layers (Leboeuf \& Mehta 1996). The rib spacing did not change when the rollers paired.

To further clarify the structure, instantaneous streamline patterns on the (two) planes of symmetry were computed by integrating the velocity vector fields; the topology of the patterns was then described in terms of critical points. With this technique, the aforementioned rollers are clearly evident. So too are 'cross ribs' which connect rollers across the symmetry plane with a spanwise spacing thrice that of the nozzle flutes. But, unlike successive ribs which evolve soon after the rollers form, cross ribs occur much later. Indeed cross ribs occur only after the rollers have undergone a spanwise perturbation whose amplitude has grown large enough to allow mirror image rollers to touch.

As the flow traverses the impingment region, it diverges about a mean stagnation streamsurface and the rollers follow suite. As they do so, the amplitude of their spanwise periodic perturbation increases. However, although the form of the perturbation initially remains unchanged, it ultimately contorts (as the rollers exit the impingment region), into what appear to be two connected, but still spanwise periodic, lobes. Meanwhile the distance between the rollers and the jet symmetry plane increases and the vorticity from which the cross ribs are composed is stretched and enhanced. Moreover, that same vorticity is seen to align with the mean strain, as does the vorticity in successive ribs. Of course as the rollers move apart they also approach the wall and at some point the cross ribs encounter and merge with wall ribs composed of vorticity of the same sign. At this juncture the production of streamwise (in the sense of the plate) vorticity due to stretching (not reorientation) is a maximum and that maximum is highest in the vicinity of, and peaks at, the symmetry plane. The demise of successive ribs on the other hand occurs away from the symmetry plane and would appear to be less important dynamically than cross ribs merging with wall ribs.

Wall ribs are evident throughout the cycle. Moreover, because they are evident both with and without (phase and temporal) locking, they would appear to have their origins in the boundary layer rather than upstream, although their lateral spacing can most definitely be determined upstream (Yokobori et al. 1977). Using other techniques, Lyell \& Huerre (1985) also conclude that wall ribs form in the boundary layer. Specifically, they determine that disturbances added to Hiemenz flow are attenuated and stabilized by concave streamline curvature, thereby excluding bifurcations to secondary flow. There are of course other instability mechanisms by which wall ribs can form in shear layers (see e.g. Benney \& Lin 1960; Wu 1993; Phillips \& Shen 1996), but none have been investigated in the context of Hiemenz flow. However whatever the origin of wall ribs, there is little doubt they are enhanced if not sustained by vorticity in cross ribs advected from upstream by the jet.

We are grateful to Mr D. Hayashi for help with the experiment, which was conducted at Keio University, and to Professors M. Maeda and R. D. Moser for helpful discussions. Support from the Ministry of Education of Japan (grant number 04555050), the Japan Society for the Promotion of Science and the US National Science Foundation (OCE-9696161, OCE-9818092) is gratefully acknowledged.

\section{REFERENCES}

Antonia, R. A., Chambers, A. J., Britz, D. \& Browne, L. W. B. 1986 Organized structures in a turbulent plane jet: toplogy and contribution to momentum and heat transport. J. Fluid Mech. 163, 365-391.

Batchelor, G. K. 1967 An Introduction to Fluid Dynamics. Cambridge University Press. 
Bell, J. H. \& Mehta, R. D. 1992 Measurements of the streamwise vortical structures in a plane mixing layer. J. Fluid Mech. 239, 213-248.

Beltaos, S. \& Rajaratnam, N. 1973 Plane turbulent impinging jets. J. Hydr. Res. 11, 29-45.

Benney, D. J. \& Lin, C. C. 1960 On the secondary motion induced by oscillations in a shear flow. Phys. Fluids 3, 656-657.

Bernal, L. P. \& Roshko, A. 1986 Streamwise vortex structure in plane mixing layers. J. Fluid Mech., 170, 499-526.

Chong, M. S., Perry, A. E. \& Cantwell, B. J. 1990 A general classification of three-dimensional flow fields. Phys. Fluids A 2, 765-777.

Crow, S. C. 1970 Stability theory for a pair of trailing vortices. AIAA J. 8, 2172-2179.

DidDen, N. \& Ho, C.-M. 1985 Unsteady separation in a boundary layer produced by an impinging jet. J. Fluid Mech. 160, 235-256.

Fox, M. D., Kurosaka, M., Hedges, L. \& Hirano, K. 1993 Influence of vortical structures on jet thermal fields. J. Fluid Mech. 255, 447-472.

GARDON, R. \& AKfirat, J. C. 1965 The role of turbulence in determining the heat-transfer characteristics of impinging jets. Intl J. Heat Mass Transfer 8, 1261-1272.

Gutmark, E., Wolfshtein, M. \& Wygnanski, I. 1978 The plane turbulent impinging jet. J. Fluid Mech. 88, 737-756.

HayaKaWa, M. \& Hussain, F. 1989 Three-dimensionality of organized structures in a plane turbulent wake. J. Fluid Mech. 206, 375-404.

Herbert, T. 1988 Secondary instability of boundary layers. Ann. Rev. Fluid Mech. 20, 487-526.

HuAnG, L. S. \& Ho, C. M. 1990 Small-scale transition in a plane mixing layer. J. Fluid Mech. 210, $475-500$.

Hunt, J. C. R., Abell, C. J., Peterka, J. A. \& Woo, H. 1978 Kinematical studies of the flows around free or surface mounted obstacles: applying topology to flow visualization. J. Fluid Mech. 86, 179-200.

Hunt, J. C. R., Wray, A. A., \& Moin, P. 1988 Eddies, stream and convergence zones in turbulent flows. Center for Turbulence Research Rep. CTR-S88, p. 193.

Hussain, A. K. M. F. 1983 Coherent structures and Incoherent turbulence. In Turbulence and Chaotic Phenomena in Fluids (ed. T. Tatsumi), pp. 453-460, North Holland.

Hussain, A. K. M. F. 1986 Coherent structures and turbulence. J. Fluid Mech. 173, 303-356.

Hussain, A. K. M. F. \& Hayakawa, M. 1987 Eduction of large-scale organized structures in a turbulent plane wake. J. Fluid Mech. 180, 193-229.

INOUE, O. 1992 Double-frequency forcing on spatially growing mixing layers. J. Fluid Mech. 234, $553-581$.

JeOng, J. \& Hussain, F. 1995 On the identification of a vortex. J. Fluid Mech. 285, 69-94.

Lasheras, J. S., Cho, J. S. \& MaXworthy, T. 1986 On the origin and evolution of streamwise vortical structure in a plane free shear layer. J. Fluid Mech. 172, 231-258.

Lasheras, J. C. \& CHOI, H. 1988 Three-dimensional instability of a plane free shear layer: an experimental study of the formation and evolution of streamwise vortices. J. Fluid Mech. 189, 53-86 (referred to herein as LC)

Leboeuf, R. L. \& Mehta, R. D. 1995 Measurements of spanwise scale change in a forced mixing layer. J. Fluid Mech. 293, 305-319.

Leboeuf, R. L. \& MehtA, R. D. 1996 Vortical structure morphology in the initial region of a forced mixing layer: roll-up and pairing. J. Fluid Mech. 315, 175-221.

Lighthill, M. J. 1963 In Laminar Boundary Layers (ed. L. Rosenhead), pp 48-88. Oxford University Press.

LOONEY, M. K., \& WaLSh, J. J. 1984 Mean-flow and turbulent characteristics of free and impinging jet flows. J. Fluid Mech. 147, 397-429.

Lugt, H. J. 1996 Introduction to Vortex Theory. Vortex Flow Press, Maryland, USA.

Lyell, J. \& Huerre, P. 1985 Linear and nonlinear stability of plane stagnation flow. J. Fluid Mech. 161, 295-312.

NygaARd, K. J. \& Glezer, A. 1991 Evolution of streamwise vortices and generation of small-scale motion in a plane mixing layer. J. Fluid Mech. 231, 257-301.

Panton, R. L. 1996 Incompressible Flow. Wiley-Interscience.

Perry, A. E. \& Fairlie, B. D. 1974 Critical points in flow patterns. Adv. Geophys. B 18, 299-315. 
Perry, A. E. \& Lim, T. T. 1978 Coherent structures in coflowing jets and wakes. J. Fluid Mech. 88, 451-463.

Perry, A. E. \& TAN, D. K. M. 1984 Simple three-dimensional vortex motions in coflowing jets and wakes. J. Fluid Mech. 141, 197-231.

Phillips, W. R. C. \& Shen, Q. 1996 On a family of wave-mean shear interactions and their instability to longitudinal vortex form. Stud. Appl. Maths 96, 143-161.

Phillips, W. R. C. \& WU, Z. 1994 On the instability of wave-catalysed longitudinal vortices in strong shear. J. Fluid Mech. 272, 235-254.

Phillips, W. R. C., Wu, Z. \& Lumley, J. L. 1996 On the formation of longitudinal vortices in turbulent boundary layers over wavy terrain. J. Fluid Mech. 326, 321-341.

Pierrehumbert, R. T. \& Widnall, S. E. 1982 The two- and three-dimensional instabilities of a spatially periodic shear layer. J. Fluid Mech. 114, 59-82.

Press, W. H., Flannery, B. P., Teukolsky, S. A. \& Vetterling, W. T. 1986 Numerical Recipes. Cambridge University Press.

Rogers, M. M. \& Moser, R. D. 1992a The three-dimensional evolution of a plane mixing layer: the Kelvin-Helmholtz rollup. J. Fluid Mech. 243, 183-226.

Rogers, M. M. \& Moser, R. D. $1992 b$ Spanwise scale selection in plane mixing layers. J. Fluid Mech. 247, 321-337.

SADEH, W. Z. \& BRAUER, H. J. 1980 A visual investigation of turbulence in stagnation flow about a circular cylinder. J. Fluid Mech. 99, 53-54.

SaKakibara, J., Hishida, K. \& Maeda, M. 1995 Three-dimensional vortex structure in the stagnation region of a forced plane impinging jet. Bull. Am. Phys. Soc. 40, 1933.

SaKakibara, J., Hishida, K. \& MAeda, M. 1997 Vortex structure and heat transfer in the stagnation region of an impinging plane jet (Simultaneous measurements of velocity and temperature fields by digital particle image velocimetry and laser-induced fluorescence). Intl J. Heat Mass Transfer 40, 3163-3176.

Sutera, S. P., Maeder, P. F. \& Kestin, J. 1963 On the sensitivity of heat transfer in the stagnationpoint boundary layer to free-stream vorticity. J. Fluid Mech. 16, 497-520.

VAN DyKe, M. 1982 An Album of Fluid Motion. Parabolic Press.

WU, X. 1993 Nonlinear temporal-spatial modulation of near planar Rayleigh waves in shear flows: formation of streamwise vortices. J. Fluid Mech. 256, 685-719.

Yokobori, S., Kasagi, N. \& Hirata, M. 1977 Characteristic behavior of turbulence in the stagnation region of a two-dimensional submerged jet impinging normally on a flat plate. Proc. Symp. Turbulent Shear Flows, Penn State (ed. F. Durst), pp. 3.17-3.25. Springer. 